Western University Scholarship@Western

1993

\title{
Destructive Interference in an Imperfectly Competitive Multi-Security Market
}

Utpal Bhattacharya

Philip J. Reny

Matthew Spiegel

Follow this and additional works at: https://ir.lib.uwo.ca/economicsresrpt

Part of the Economics Commons

Citation of this paper:

Bhattacharya, Utpal, Philip J. Reny, Matthew Spiegel. "Destructive Interference in an Imperfectly Competitive Multi-Security Market." Department of Economics Research Reports, 9318. London, ON: Department of Economics, University of Western Ontario (1993). 


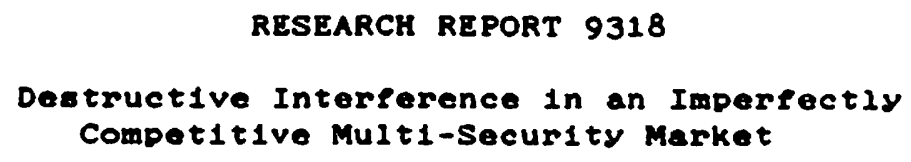

by

Utpel Bhettacharya Phil1p J. Reny

Metthew Splegel

September 1993

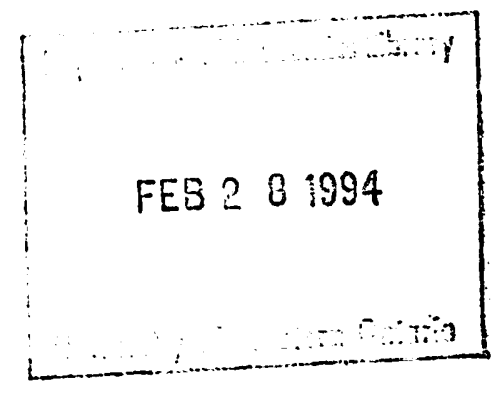

\author{
Depertment of Economice \\ Sociel Science Centre \\ Un1versity of Weetern Ontar10 \\ London, Ontar10, CANADA \\ N6A $5 C 2$
}




\title{
Destructive Interference in an Imperfectly \\ Competitive Multi-Security Market
}

\author{
by \\ Utpal Bhattacharya ${ }^{1}$ \\ Philip J. Reny ${ }^{2}$ \\ and \\ Matthew Spicgel ${ }^{3}$ \\ March, 1991 \\ Revised: September 1993
}

'University of lowa, College of Business Administration, lowa City, IA 52242. Ph: (319) 335-1239.

${ }^{2}$ University of Westem Ontario, Department of Economics, London, Ontario, Canada N6A 5C2. Ph: (519) 6613492. Support from the Social Sciences and Humanities Research Council of Canada is gratefully acknowledged. Some of this research was carried out while this author was visiting the Department of Economics at the University of Wisconsin, Madison.

${ }^{3}$ University of Califomia-Berkeley, Haas School of Business, 350 Barrows Hall, Berkeley, CA 94720. Ph: (510) 642-3421.

*We wish to thank Gur Huberman and Arthur Robson for helpful discussions, and Martin Hellwig whose comments ultimatcly led us to take a signalling game approach. We also thank S. Viswanathan for his comments at the mcetings of the Westem Finance Association. 


\section{Destructive Interference}

Philip Reny, University of Westem Ontario, Department of Economics, London, Ontario, Canada N6A 5C2. Ph: (519) 679-2111 ext. 5484. 
B1 - Bhattacharya, Utpal; Reny, Philip and Spiegel, Matthew

B2 - Destructive Interference in an Imperfectly Competitive Multi-Security Market

C2 - A general equilibrium framework is employed to illustrate how the advent of trading in new securities, about which an "insider" has private information, might cause a collapse of prevailing security markets. Roughly, securities that allow the insider to hedge his portfolio risk interfere with one other, and in some cases, so much so that these securities cannot simultaneously trade in any equilibrium.

B6 - J. Econ. Theory.

B7 -

B8 -

C4 -

B4 - University of Iowa, College of Business Administration, Iowa City, IA 52242; University of Westem Ontario, Department of Economics, London, Ontario, Canada N6A 5C2; Haas School of Business, 350 Barrows Hall, University of Califomia-Berkeley, Berkeley, CA 94720.

Joumal of Economic Literature Classification Numbers(s): G14, G12, C72 


\section{INTRODUCTION}

In 1970, Akerlof [1] convincingly argued that the presence of asymmetric information can profoundly affect equilibrium trading patterns. In the context of financial markets with an informed trader possessing "inside information", Akerlof's ideas are pursued by the consideration of so-called rational expectations equilibria. As we are now well aware, under rational expectations the presence of an informed trader on one side of a market can preclude the possibility of any trade taking place simply because those on the other side know that they will be taken advantage of.

Holding to the rational expectations hypothesis, and within the context of a multi-security model of financial markets, the present paper provides conditions under which a given collection of securities cannot simultaneously trade due to the presence of insider trading. In particular, if the insider's trading activity is due largely to his possession of private information on future security values, then no trade occurs. The analysis provides a complete characterization of precisely when the insider's risk and information characteristics render trade impossible. Moreover, this characterization provides results on the effect of attempting to introduce new securities into the market.

There are by now several papers examining the question of market breakdowns in a single security setting, including Glosten [12], Leach and Madhavan [20], Bhattacharya and Spiegel [6], and Hellwig [15]. However, the present setting allows for any number of securities, and so it is capable of addressing issues that cannot even be formulated in a single security environment. For instance, suppose the market is in cquilibrium and a ncw security is introduced. What impact might this havc? In particular, can an equilibrium be sustained in which this larger collection of securities simultaneously trade? Altematively, suppose that a given collection of securities cannot simultaneously trade in equilibrium. (The singlesecurity literature has already demonstrated that this can be the case.) Can an equilibrium with trade be restored by adding an appropriately designed new security?

Our results provide a negative answer to the second question (Theorem 5.2). There is no security 
which, when added to the market, can restore equilibrium. Regarding the first question, we find that adding a security to a market that is in equilibrium may lead to a collection of securities that is incompatible with equilibrium. Consequently, the only way to restore equilibrium after such an addition is to close the market in the new security or to close some other security market(s) (remember, opening new security markets cannot help). Since our model does not include any dynamics, we cannot formally address which of these closures will take place, only that some closure must take place. Generally, whenever a set of sccurity markets cannot open simultaneously, we'll say that they destructively interfere with one another.

Loosely, destructive interference occurs whenever payoffs from the set of currently trading securities are highly correlated. This leads to a number of practical implications. For instance, our results suggest that the underlying correlation among the unknown factors influencing the retums of an individual project may determine whether an owner-manager must ultimately issue one security for a conglomerate or whether he can issue individual securities for the different units. Similarly, derivative securities, whose payoffs are typically highly correlated with the payoff of the primitive assets, may well eliminate the market for the primitive asset if the initial amount of insider trading in the primitive asset is sufficiently large. Stretching the model somewhat, this may explain why deep in the money options do not trade. Deep in the money options have payoffs that are almost perfectly correlated with the underlying stock. Although our assumptions do not precisely fit this case (our security payoffs are linear) our results suggest that in equilibrium either the option or the stock can trade but not both.

As in Admati [2], where the model of Hellwig [14] is generalized to include multiple securities, we find that Giffen securities can arise. ${ }^{1}$ This contrasts with the results of Caballe and Krishnan [7] in their generalization of Kyle [19]. However, our model differs significantly from Kyle's [19] and Caballe and Krishnan's [7] in an important respect. Like the papers of Bray [5], Ausubel [3], Gale and Hellwig [11], Glosten [12], Laffont and Maskin [21], and Bhattacharya and Spiegel [6], noise traders are absent 
from our model. However, prices remain only partially revealing because our insider has both a hedging and an informational motive for trade. The absence of noise traders is crucial and it is precisely why we do obtain Akerlof-like market failures in the presence of asymmetric information. Because all of our agents are rational, those agents without inside information will rationally choose not to trade when the insider's willingness to trade is largely due to his private information. In models involving noise traders however, the market can never collapse to a no trade equilibrium regardless of the insider's informational advantage simply because noise traders are assumed to trade in spite of any losses that they might incur as a result.

To date, most of the literature on rational expectations equilibria in financial markets with asymmetric information has restricted attention to the linear equilibrium. This has largely been for reasons of tractability. Since our results often focus on the absence of equilibria involving trade in all (or some) securities, we are compelled to broaden the search for equilibria beyond those that are linear. Like the analyses of Glosten [12], Ausubel [3], Laffont and Maskin [21], and Bhattacharya and Spiegel [6] we do this. In fact, we go much further. We impose no restrictions on the equilibrium price schedule. Our only requirement is that in equilibrium the outsider's beliefs about the insider's trading motives satisfy Cho and Krep's [8] D1 criterion. ${ }^{2}$ To our knowledge this is the first attempt in the normal-exponential noisy rational expectations literature to do away with continuity restrictions on the equilibrium price schedule.

Remarkably, the analysis shows that the linear equilibria are, in a sense, focal. Fixing a collection of securities to be traded, the existence of a linear equilibrium involving trade in these securities is both necessary and sufficient for the existence of any equilibrium at all involving trade in these securities.

The paper proceeds as follows. The model is described in section 2 . In section 3 we define an equilibrium in which all potentially tradable securities are in fact tradable. Section 4 is devoted to the existence of such an equilibrium. Subsection 4.1 provides a characterization for the existence of a linear such equilibrium, and 4.2 shows that an equilibrium in which all potentially tradable securities are tradable 
exists if and only if such a linear equilibrium exists. Section 5 considers the more general question of the existence of equilibria in which only some subset of the potentially tradable securities is tradable. Section 6 concludes.

\section{THE MODEL}

The basic setting is an $\mathrm{n}$ security version of the single security model found in Bhattacharya and Spiegel [6]. Traders are divided into two groups, each having negative exponential utility. The first consists of a single large trader (the "insider") who has private information about the securities within the economy. His strictly positive risk aversion parameter is denoted by $\theta$. A continuum of small traders ("outsiders"), indexed by $\alpha \in[0,1]$, make up the second group. Their distribution over the interval $[0,1]$ is given by the atomless measure $v$, which we normalize so that $v([0,1])=1$. Each outsider has a strictly positive risk aversion parameter, $\phi$.

There are n potentially tradable securities and one (always tradable) risk-free bond. Among the collection of potentially tradable securities only some subset, to be determined in equilibrium, can actually trade. Those that remain untraded may, for instance, be interpreted as securities not presently in the market since they have not yet been "designed", or simply as securities that are not yet publicly traded. Here we do not concem ourselves with the incentives involved in either designing new securities or in some other way introducing new securities into the market. Rather, we concentrate primarily on whether a given collection of securities, whatever their origin, can remain tradable as a group in equilibrium.

Quantities of the $n$ potentially tradable securities are denoted by $S_{1}, \ldots S_{n}$ and quantities of the riskless bond (or debt) by D. The endowment of outsider $\alpha \in[0,1]$ is $\left(\overline{\mathbf{D}}(\alpha), \overline{\mathbf{S}}_{1}(\alpha), \ldots, \overline{\mathbf{S}}_{n}(\alpha)\right)$ and it is assumed that $\overline{\mathbf{D}}:[0,1] \rightarrow \mathbf{R}$ is Borel measurable as is $\bar{S}_{i}:[0,1] \rightarrow \mathbf{R}$ for each $i=0,1, \ldots, n{ }^{3}$ Moreover, we normalize the supply of every security and the bond to unity so that the insider's endowment of the $i^{\text {th }}$ 
security is $\bar{S}_{\mathrm{i}}$, where $\overline{\mathrm{S}}_{\mathrm{i}} \equiv 1-\int_{0}^{1} \bar{S}_{i}(\alpha) d v$, and his bond endowment is $\overline{\mathrm{D}} \equiv 1-\int_{0}^{1} \overline{\mathrm{D}}(\alpha) d v$. In addition, the insider

is randomly endowed with a set of $\mathbf{n}$ untradable assets. These $\mathbf{n}$ random quantities are denoted by $\mathbf{W}_{1}, \ldots, \mathbf{W}_{\mathrm{n}}$. This collection, which is always untradable, may include items such as human capital, and real estate. Note that it is not necessary to think of the insider as holding n one-dimensional untradable assets. He may instead hold a smaller number of untraded multi-dimensional ones. The realized quantities of the insider's untraded assets form part of his private information. In the model, outsiders know only that $\mathbf{W}$ $=\left(W_{1}, \ldots, W_{n}\right)$ is normally distributed with mean zero and covariance matrix $\Sigma_{w}$.

Let us fix, for the moment, a subset of the set of potentially tradable securities. Suppose these securities' indices are $\{1,2, \ldots, \mathrm{k}\}$, and that they constitute the set of tradable securities. All agents must therefore retain their endowed amounts of the untradable securities $k+1$ through $n$. When an agent wishes to trade a (tradable) security, he must do so through a "Walrasian market-maker" who, in equilibrium, quotes market-clearing prices. ${ }^{4}$

Formally, the market-maker is simply a mapping from demands made by the insider to security prices. That is, for every vector of security demands, $S^{I}$, submitted by the insider, the market-maker (price schedule) provides prices $P\left(S^{I}\right)=\left(P_{1}\left(S^{I}\right), \ldots, P_{k}\left(S^{I}\right)\right)$, for the $k$ tradable securities. The price and payoff of the bond is normalized to unity. It is common knowledge that the future value of the $i^{\text {th }}$ security is given by $\varepsilon_{i}+\eta_{i}$ for $i=1,2, \ldots n$, where $\left(\varepsilon_{1}, \ldots, \varepsilon_{n}\right)$ and $\left(\eta_{1}, \ldots, \eta_{n}\right)$ are independent random vectors normally distributed with means zero and covariance matrices $\Sigma_{\varepsilon}$ and $\Sigma_{\eta}$ respectively. The mean zero assumptions serve only to reduce the notational burden. To simplify the analysis, we assume that the future value of the insider's $i^{\text {th }}$ untraded asset $W_{i}$, is also $\varepsilon_{i}+\eta_{i}$ for $i=1,2, \ldots, n$.

Note that the insider receives private information on all $\mathrm{n}$ securities, even if not all are tradable. This allows us to investigate the effect of introducing new securities into the market about which the 
insider has private information, without discussing incentives to acquire private information which might well be related to those securities that are actually tradable. The insider's private information is purely exogenous, and does not change with the number or make-up of tradable securities.

Given the set of tradable securities $\{1,2, \ldots, \mathbf{k}\}$ and the market-maker's price schedule $\mathbf{P}: \mathbf{R}^{\mathbf{k}} \rightarrow \mathbf{R}^{\mathbf{k}}$, both of which are common knowledge among all traders, the order of events is as follows: First, nature chooses the values of the random vectors $\varepsilon=\left(\varepsilon_{1}, \ldots, \varepsilon_{n}\right), \eta=\left(\eta_{1}, \ldots, \eta_{n}\right)$ and $W=\left(W_{1}, \ldots, W_{n}\right)$ independently according to their distributions. Second, the insider is informed of both $w$ and $\varepsilon$, but not $\eta$. The insider then submits a demand for securities 1 through $\mathbf{k}$ to the market-maker. Third, the market-maker consults the price schedule and fixes the price at which all tradable securities trade (and hence determines the insider's bond demand through his budget constraint). Fourth, and finally, outsiders are informed of these prices (but not of $W, \varepsilon$, or $\eta$ ) and thereafter submit their demands for tradable securities (their bond demands again being determined by their budget constraints).

Notice that one can interpret the random vectors $\eta$ and $\varepsilon$ as economy-wide uncertainty and outsider-specific uncertainty about the future value of securities $1,2, \ldots . \mathrm{n}$. We assume throughout that the variance-covariance matrices $\Sigma_{\varepsilon}, \Sigma_{\eta}$ and $\Sigma_{w}$ are of full rank and therefore positive definite.

The description of an equilibrium shall provide both the collection of tradable securities and the market-maker's price-schedule. If it were not for the insider's private information about the security payoffs, any subset of the securities could trade in some equilibrium. As we shall see however, the insider's presence profoundly affects the possible equilibrium market structures. Under certain conditions some securities simply cannot simultaneously trade. ${ }^{5}$

Since we do not rule it out, there is always a trivial equilibrium in which none of the potentially tradable securities are actually tradable. In this equilibrium all traders simply consume their endowments, which is optimal since there is no possibility of trade. Hence, the existence of an equilibrium, since the set of tradable securities is part of its description, never presents a difficulty. Our main objective is to 
determine, for any subset of the set of potentially tradable securities, precisely when the insider's information allows these to be tradable in some equilibrium. Thus, the analysis below proceeds by fixing a collection of securities, deeming precisely these to be tradable, and then determines whether or not the market-maker can set prices for these securities so that markets clear. We begin by supposing that all potentially tradable securities are tradable.

\section{EQUILIBRIUM}

Throughout this section, as well as Section 4, we shall suppose that all securities are tradable. The analysis when a strict subset of the set of potentially tradable securities is tradable is virtually identical and is presented in Section 5. So, for the time being, the market structure is constrained so that all $\mathbf{n}$ securities are tradable. We wish to know when this market structure is compatible with equilibrium.

Given any market-maker price schedule $\mathbf{P}: \mathbf{R}^{\mathfrak{n}} \rightarrow \mathbf{R}^{\mathbf{n}}$, the insider will, for every realization, $\mathbf{W}$, of $W$ and $\varepsilon$ of $\varepsilon$, demand a vector of securities $S_{1}, S_{2}, \ldots, S_{n}$ and an amount of the bond which maximizes his expected utility subject to his budget constraint. That is (making use of the exponential utility assumption), the insider chooses $\left(D^{I}, S^{I}\right) \in R^{n+1}$ to maximize

$$
V_{I}\left(D^{I}, S^{I} \mid W, \varepsilon\right)=\left(S^{I}+W\right)^{T} \varepsilon+D^{I}-.5 \theta\left(S^{I}+W\right)^{T} \Sigma_{\eta}\left(S^{I}+W\right)
$$

subject to

$$
\left[S^{I}-\bar{S}\right]^{T} \mathbf{P}\left(S^{I}\right)+D^{I}-\bar{D}=0
$$

Substituting (2) into (1) renders this equivalent to choosing $S \in \mathbf{R}^{\mathbf{n}}$ to maximize

$$
\mathrm{V}_{\mathrm{I}}\left(\mathbf{S}^{\mathrm{I}} \mid \tau\right) \equiv\left(\mathrm{S}^{\mathrm{I}}\right)^{\mathrm{T}} \tau-\left(\mathrm{S}^{\mathrm{I}}-\overline{\mathrm{S}}\right)^{\mathrm{T}} \mathbf{P}\left(\mathbf{S}^{\mathrm{I}}\right)-.5 \theta\left(\mathrm{S}^{\mathrm{I}}\right)^{\mathrm{T}} \Sigma_{\eta} \mathrm{S}^{\mathrm{I}}
$$

where $\tau \equiv \varepsilon-\theta \Sigma_{\eta} W$. 
As exhibited by (3), the insider's preferences depend only on $\varepsilon$ and $W$ in so far as $\tau$ is affected. Consequently, for each $\varepsilon$ and $W$, the variable $\tau$ completely determines the insider's preferences and we shall therefore refer to $\tau$ as the insider's "type". The random variable generating the insider's type will be denoted by $\tau$ and is defined by $\tau \equiv \varepsilon-\theta \Sigma_{\mathrm{n}} \mathbf{W}$. It now becomes natural to model the entire scenario as a signalling game. Each insider (sender) type demands some quantity of each security. Given the price schedule, this determines each security's price and these prices are the signals which ultimately reach the outsiders (receivers). The outsiders use the prices (signals) to make inferences about the insider's type which leads to inferences about $\varepsilon$, and the future value of the securities' payoffs. The outsiders then place their demands for each security. In equilibrium, all security markets must clear.

Consequently, a strategy for the insider is a mapping $\mathbf{S}^{\mathrm{I}}: \mathbf{R}^{\mathrm{n}} \rightarrow \mathbf{R}^{\mathrm{n}}$ taking values of his type, $\tau$, into a demand for each of the $n$ securities, while a strategy for each outsider $\alpha \in[0,1]$ is a mapping $\mathbf{S}^{\alpha}: \mathbf{R}^{\mathrm{n}} \rightarrow \mathbf{R}^{\mathrm{n}}$ taking the observed prices into a demand for each security. Note that the present model is not a game in the strict sense because the price schedule, which in equilibrium must equate demand and supply in each security market, although endogenous, is not chosen by any maximizing agent within the model. Thus, we continue to employ the standard general equilibrium view of prices, while attempting to exploit as much as possible the insights available from the signalling-game literature.

In many signalling models the need arises to discipline the inferences made by the receiver upon the receipt of a signal that is unsent in equilibrium. ${ }^{6}$ The present model is no exception. Indeed, we shall discipline the outsiders' inferences in a by now rather standard fashion. They must satisfy Cho and Kreps' [8] D1 criterion.

We proceed informally to outline the effect of the D1 criterion in our context. First, note that in equilibriun, the price schedule must be one to one. Otherwise, for two distinct security demands of the insider, the security demands of the outsiders will be the same (the latter can depend only on the observed price vector). But then for one of these demands, the securities market will not clear. 
Suppose now that the outsiders observe the vector of security prices, $P_{0}$, but that $P_{0}$ can never arise if the insider follows his equilibrium strategy. Because the equilibrium price schedule is one to one, the outsiders can invert it to determine the out-of-equilibrium demand submitted by the insider. Call this demand $S_{0}$. Since no insider-type demands $S_{0}$ in equilibrium, the outsiders cannot employ Bayes' rule to form an inference about which insider-type(s) might have submitted such a demand. The D1 criterion suggests a procedure for disciplining the outsiders' inferences. The idea is to insist that the outsiders provide a "reasonable" explanation for the deviation. Indeed, the outsiders are asked to take the vicw that the insider is, by deviating, attempting to raise his payoff above what he would obtain in equilibrium.

Now if the insider submits the out-of-equilibrium demand, $S_{0}$, in order to raise his payoff above what he could obtain in equilibrium, then he must believe that the market-maker will respond with a more favorable price than that dictated by the equilibrium price schedule for $S_{0}$. Let this then serve as the outsiders' explanation for the insider's deviation.

But this explanation allows the outsiders to isolate those insider-types who are most likely to have deviated. Indeed, this is the strength of the D1 criterion and it works as follows. Suppose a subset, T, of insider-types possesses the following property:

Whenever a potential price response to $S_{0}$ provides some insider-type not in $T$ with an incentive (perhaps only weak) to deviate to $S_{0}$, the same price response provides every member of $T$ with a strict incentive to deviate to $S_{0}$.

Under these circumstances we will call $\mathrm{T}$ a prime suspect subset of insider types for $S_{0}$ (in the sense that the types in $\mathrm{T}$ are deemed most likely to have deviated). The D1 criterion restricts the outsiders' beliefs to be concentrated on the set $T^{8}$ We now formally describe an equilibrium.

An equilibrium in securities $\{1,2, \ldots, n\}$ is a tuple $\left(\mathbf{P}(\cdot), \mathbf{S}^{\mathbf{I}}(\cdot),\left(\mathbf{S}^{\alpha}(\cdot)\right)_{\alpha \in[0,1]}\right)$, where $\mathbf{P}: \mathbf{R}^{\mathbf{n}} \rightarrow \mathbf{R}^{\mathbf{n}}$, $S^{I}: R^{n} \rightarrow R^{n}, S^{\alpha}: R^{n} \rightarrow R^{n}$, satisfy for every insider type $\tau=\varepsilon-\theta \Sigma_{\eta} W$, every outsider $\alpha \in[0,1]$, and every $\hat{S}=\mathbf{S}^{\mathbf{I}}(\tau)$ 
(i) $\hat{\mathrm{S}}$ maximizes the insider's expected utility given his type $\tau$ and the price schedule $\mathbf{P}(\cdot)$,

(ii) $\quad \mathbf{S}^{\mathbf{\alpha}}(\mathbf{P}(\hat{S}))$ maximizes outsider $\alpha$ 's expected utility given the price schedule $\mathbf{P}(\cdot)$, the insider's strategy $S^{\mathrm{I}}(\cdot$, and $\alpha$ 's Bayes rule induced beliefs about the insider's type $\tau$, and therefore $\varepsilon$,

(iii) The outsider's beliefs satisfy Cho and Kreps' D1 criterion. That is, if $S \neq S^{I}(\tau)$ for all $\tau^{\prime}$, and $T$ is a prime suspect subset of insider types for $S$, then $\mathfrak{I}(\mathbf{P}(\mathbf{S})) \subseteq T$, where $\mathfrak{I}(\mathrm{P})$ denotes the support of the outsiders' beliefs about the insider's type, conditional upon observing the price vector $P{ }^{9}$

(iv) $\quad \mathbf{S}^{\mathrm{l}}(\cdot)$ is Borel measurable as is $\mathbf{S}^{\alpha}(\mathbf{P}(\mathbf{S}))$ in $\alpha$, for every $S$,

(v) $\int_{0}^{1} S^{\alpha}(P(S)) d v=1-S$, for every $S$, where 1 is an $n \times 1$ vector of 1 's.

Conditions (i) and (ii) ensure that in equilibrium the insider and the outsiders are making utility maximizing decisions and that the outsiders, when possible, employ Bayes' rule to make inferences about the insider's private information. Condition (iii) disciplines the outsider's beliefs to conform to the D1 criterion. Condition (iv) is simply a regularity requirement. Finally, condition (v) asks that for any security demand made by the insider, the equilibrium prices set by the market-maker clear all markets.

\section{EXISTENCE OF AN EQUILIBRIUM}

\subsection{THE LINEAR EQUILIBRIUM}

As we shall later see, the existence of any equilibrium in securities $\{1,2, \ldots, n\}$ rests on the existence of an equilibrium involving a linear price schedule or simply, a linear equilibrium. We therefore consider the conditions leading to an equilibrium in which $\mathbf{P}\left(S^{\mathbf{I}}\right)=A S^{\mathrm{I}}+\mathrm{b}$ for some $n \times n$ matrix $A$ and $n \times 1$ vector $b$. Recall from the discussion in the previous section that $P(\cdot)$ must be one to one. Consequently, A must be nonsingular. We begin by deriving the insider's optimal strategy. Since the maximization of (3) is 
unconstrained, we obtain the following first-order condition for insider-type $\tau$ by differentiating with respect to $S^{I}$ :

$$
\tau-A S^{I}-b-A^{T}\left(S^{I}-\bar{S}\right)-\theta \Sigma_{\eta} S^{I}=0
$$

In light of this, consider the information about $\varepsilon$ that can be obtained in equilibrium by an outsider upon observing the equilibrium price vector. Since $A$ is invertible, the observed price vector can be used to determine the insider's demand, $S^{I}$. The outsiders can then solve (4) for the insider's type $\tau$, so that the realized values of $\varepsilon$ and $W$ must satisfy $\tau=\varepsilon-\theta \Sigma_{\eta} W$. Since the insider's strategy depends only upon his type $\tau$, the outsiders cannot refine their information about $\varepsilon$ any further. Consequently, because $\varepsilon$ and $\mathbf{W}$ are independently and normally distributed with zero means and covariance matrices $\Sigma_{\varepsilon}$ and $\Sigma_{W}$ respectively, a standard application of Bayesian updating yields that the outsiders' resulting conditional distribution of $\varepsilon$ is normal with mean $U \tau$ and covariance matrix $\Sigma_{\varepsilon \mid \tau} \equiv\left[\Sigma_{\varepsilon}^{-1}+\left(\theta^{2} \Sigma_{\eta} \Sigma_{W} \Sigma_{\eta}\right)^{-1}\right]^{-1}$, where $\mathrm{U}=\Sigma_{\varepsilon}\left[\theta^{2} \Sigma_{\eta} \Sigma_{W} \Sigma_{\eta}+\Sigma_{\varepsilon}\right]^{-1}$

Consider now an outsider $\alpha \in[0,1]$ who has observed the equilibrium prices and deduced the insider's type $\tau$. Since his utility is exponential, he chooses a vector of securities $S^{\alpha}$ and an amount of the bond $D^{\alpha}$ to maximize (after updating his beliefs about $\varepsilon$ as described above):

$\left(S^{\alpha}\right)^{T} U_{\tau+D^{\alpha}}-.5 \phi\left(S^{\alpha}\right)^{T} \Sigma_{0} S^{\alpha}$

subject to the budget constraint $\left(S^{\alpha}-\bar{S}^{\alpha}\right)^{T}\left(A S^{1}+b\right)+\left(D^{\alpha}-\bar{D}^{\alpha}\right)=0$

where $U \tau$ is the conditional mean of the future value of the risky securities, and $\Sigma_{0}=\Sigma_{\varepsilon \mid \tau}+\Sigma_{\eta}$ is the corresponding conditional covariance matrix. But this is equivalent to choosing $S^{\alpha}$ to maximize: $V_{0}\left(S^{\alpha}\right) \equiv\left(S^{\alpha}\right)^{T} U \tau-\left(S^{\alpha}-\bar{S}^{\alpha}\right)^{T}\left(A S^{I}+b\right)-.5 \phi\left(S^{\alpha}\right)^{T} \Sigma_{0} S^{\alpha}$ 
The relevant first-order condition is:

$$
\mathrm{U} \tau-\mathrm{A} S^{\mathrm{I}}-\mathrm{b}-\phi \Sigma_{\mathrm{o}} S^{\alpha}=0
$$

Therefore, if $\left(P()^{\cdot}, \mathbf{S}^{\mathrm{I}}\left({ }^{\cdot}\right), \mathbf{S}^{\alpha}()_{\alpha \in[0,1]}\right)$ constitutes a linear equilibrium, we must have, using (4), (5) and the market-clearing condition ( $v$ ), for every $\tau$ :

$$
\begin{aligned}
& \tau-A S^{I}(\tau)-b-A^{T}\left(S^{I}(\tau)-\bar{S}\right)-\theta \Sigma_{\eta} S^{I}(\tau)=0, \\
& U \tau-A S^{I}(\tau)-b-\phi \Sigma_{0} S^{\alpha}\left(S^{I}(\tau)\right)=0, \\
& \int_{0}^{1} S^{\alpha}\left(S^{I}(\tau)\right) d v=1-S^{I}(\tau),
\end{aligned}
$$

where we"ve written the outsider's strategy $\mathbf{S}^{\alpha}: \mathbf{R}^{\mathrm{n}} \rightarrow \mathbf{R}^{\mathrm{n}}$ as a function of the insider's demand rather than the observed price vector. This is without loss since any equilibrium price schedule must be one to one. Eqs. (6), (7), and (8) are the insider's first-order conditions, the outsiders' first-order conditions, and the market-clearing constraint respectively. Integrating (7) over $\alpha$, using $v$, and combining the result with (6) and (8) yields

$$
\begin{aligned}
& -A S^{I}(\tau)-b-A^{T}\left(S^{I}(\tau)-\bar{S}\right)-\theta \Sigma_{\eta} S^{I}(\tau) \\
& =U^{-1}\left[-A S^{I}(\tau)-b-\phi \Sigma_{0}\left(1-S^{I}(\tau)\right)\right],
\end{aligned}
$$

for every $\tau$.

At this point it is helpful to simplify the notation by dropping the superscript "I" on the insider's strategy and denote it simply as $S(\tau)=\left(S_{1}(\tau), \ldots, S_{n}(\tau)\right)$. This should cause no confusion as we no longer require an explicit reference to the outsiders' strategies. Thus, rewrite (9) as:

$$
-A S-b-A^{T}(S-\bar{S})-\theta \Sigma_{\eta} S=U^{-1}\left[-A S-b-\phi \Sigma_{0}(1-S)\right],
$$


for every $\tau$ and $S=S(\tau)$.

From the insider's first-order condition (6), for every $\tau$ the insider's optimal demand vector, $S(\tau)$, must satisfy:

$$
\left(A+A^{T}+\theta \Sigma_{\eta}\right) S(\tau)=\tau+A^{T} S-b
$$

Since by varying $\tau$, the range of the right-hand side of (11) is $\mathbf{R}^{\mathbf{n}}$, the same must be true of the left-hand side. We conclude that in a linear equilibrium $A+A^{T}+\theta \Sigma_{\eta}$ is nonsingular and that for every $\tau$ :

$$
S(\tau)=\left(A+A^{T}+\theta \Sigma_{\eta}\right)^{-1}\left(\tau+A^{T} S-b\right)
$$

Thus, by (12), every $S$ is chosen in a linear equilibrium by some insider type, and so (10) must hold for every S. But now note that the left-hand side of (10) differs only by $\tau$ from the derivative of the insider's objective with respect to $S^{T}$. We therefore obtain the necessary second-order condition by differentiating (10) with respect to $S$ (which is valid since (10) holds for every $S$ ). By doing so we obtain:

$$
\begin{aligned}
\frac{\partial^{2} V_{I}(S(\tau) \mid \tau)}{\partial S \partial S^{T}} & =-\left[A+A^{T}+\theta \Sigma_{\eta}\right] \\
& =U^{-1}\left[\phi \Sigma_{0}-A\right]
\end{aligned}
$$

where the first equality follows from the definition of $V_{1}(\cdot)$ and the second follows from $(10)$.

A necessary condition for $P(\cdot)$ and $S(\cdot)$ to form part of an equilibrium is that $\partial^{2} V_{1}(S(\tau) \mid \tau) / \partial S \partial S^{T}$ be negative semidefinite for every $\tau$. Indeed, since $A+A^{T}+\theta \Sigma_{\eta}$ must be nonsingular, $A+A^{T}+\theta \Sigma_{\eta}$ must then be positive definite. Moreover, this condition, in addition to $A$ being nonsingular and satisfying (13), is also sufficient to guarantee the existence of an appropriate b so that the resulting $P(\cdot)$ and $S(\cdot)$ form part of a linear equilibrium. ${ }^{10}$ Indeed, under these conditions the insider's 
optimization problem is strictly concave. Since the outsiders' problems are always strictly concave, solutions are characterized by their respective first-order conditions which (10) ensures are satisfied. In addition, (10) guarantees that markets clear. Lastly, because every $S$ is chosen in a linear equilibrium by some insicler-type, the D1 criterion is vacuously satisfied.

Thus, a necessary and sufficient condition for the existence of an equilibrium in securities $\{1,2, \ldots, n\}$ having a linear price schedule $P(S)=A S+b$ is that the solution, $A$, to the equation

$$
A+A^{T}+\theta \Sigma_{\eta}=U^{-1}\left(A-\phi \Sigma_{0}\right)
$$

be nonsingular, and that the matrix defined by both sides of (14) be positive definite. Under what conditions on the parameters $\theta, \Sigma_{\eta}, \Sigma_{w}, \Sigma_{\varepsilon}$, and $\phi$ will these requirements be met? The answer lies in the induced eigenvalues of $U$ and it is expressed in the following lemma. ${ }^{11}$

Lemma 4.1: Except possibly for a closed set of parameter values having Lebesgue measure zero, there is a solution, A, to (14) which is nonsingular and which renders both sides of (14) positive definite, if and only if every eigenvalue of $U$ is less than $1 / 2$.

\section{Proof: See Appendix A.}

Consequently, we have proven the following theorem which characterizes the existence of a linear equilibrium in securities $\{1, \ldots, n\}$. 
Theorem 4.2: Except possibly for a closed set of parameter values $\left(\theta, \phi, \Sigma_{\eta}, \Sigma_{w}, \Sigma_{\varepsilon}\right)$ having Lebesgue measure zero, there is a linear equilibrium in securities $\{1,2, \ldots, n\}$ if and only if every eigenvalue of the outsider's update matrix $U$ is less than 1/2.

Remark: Theorem 4.2 has content since the set of parameter values for which the eigenvalues of $U$ are all less than one-half is open and nonempty.

The intuition behind Theorem 4.2 is best captured by constructing appropriate measures of the insider's motives for trade. An equilibrium will fail to exist when the insider's motive for trade is based largely on his desire to exploit his private information rather than his desire to hedge against the uncertainty associated with the future value of his untradable assets $\mathbf{W}_{1}, \ldots, \mathbf{W}_{n} \cdot$ Let us consider first the case in which there is but a single potentially tradable security in the economy which is, in fact, tradable. Its future price is then $\eta+\varepsilon$ Let $\sigma_{\varepsilon}^{2}$, $\sigma_{\eta}^{2}$ and $\sigma_{w}^{2}$ denote the variances of $\varepsilon, \eta$, and $w$, respectively. In equilibrium, the insider chooses his demand for the single security based on the value of $\tau=\varepsilon-\theta \sigma_{\eta}^{2} w$. Moreover, it is the relative sizes of $\varepsilon$ and $\theta \sigma_{\eta}^{2} w$ that determine whether the insider's motive for trade is an informational or a hedging one. Now, from an outsider's point of view, both $\varepsilon$ and $\theta \sigma_{\eta}^{2} w$ are unknown. However, outsiders can deduce the value of $\tau=\varepsilon-\theta \sigma_{\eta}^{2} w$, in equilibrium. Now, given $\tau=\tau$ the expected values of $\varepsilon$ and $\theta \sigma_{\eta}^{2} w$, are $\left[\sigma_{\varepsilon}^{2} /\left(\sigma_{\varepsilon}^{2}+\theta^{2} \sigma_{\eta}^{4} \sigma_{w}^{2}\right)\right] \tau$ and $\left[\theta^{2} \sigma_{\eta}^{4} \sigma_{w}^{2} /\left(\sigma_{\varepsilon}^{2}+\theta^{2} \sigma_{\eta}^{4} \sigma_{w}^{2}\right)\right] \tau$ respectively. Thus, from an outsider's point of view the insider's motive for trade is relatively informational when the absolute value of the former exceeds that of the latter. But this is equivalent to the condition $\sigma_{\varepsilon}^{2}>\theta^{2} \sigma_{\eta}^{4} \sigma_{w}^{2}$

Suppose now that instead of their being but one security, there is a single mutual fund, consisting of fixed proportions of securities $S_{1}, \ldots, S_{n}$ given by the $n \times 1$ vector $x$, and that this mutual fund is the only potentially tradable "security" and is in fact actually tradable. The insider chooses his demand for this mutual fund based on the value of $x^{T} \tau=x^{T} \varepsilon-x^{T} \theta \Sigma_{\eta} W$. Carrying out precisely the same analysis as 
above yields that from an outsider's point of view, the insider's motive for trade in this mutual fund will be relatively informational when the absolute value of the conditional expectation of $x^{T} \varepsilon$ exceeds that of $x^{T} \theta \Sigma_{\eta} W$. But this is equivalent to the condition $x^{T} \Sigma_{\varepsilon} x>x^{T} \theta^{2} \Sigma_{\eta} \Sigma_{w} \Sigma_{\eta} x$.

Finally, consider the multi-security case. If there is any mutual fund, $x$, such that the insider's motive for trade in that mutual fund is relatively informational (i.e. $x^{T} \Sigma_{\varepsilon} x>x^{T} \theta^{2} \Sigma_{\eta} \Sigma_{W} \Sigma_{\eta} x$ ) then an equilibriurn will fail to exist since the insider and outsiders will wish to take the same side of any trade in this fund. Hence, there is no equilibrium when some mutual fund, $x$, satisfies $x^{T} \Sigma_{\varepsilon} x>x^{T} \theta^{2} \Sigma_{\eta} \Sigma_{w} \Sigma_{\eta} x$, or equivalently when the maximum value, choosing $x$ not zero, of $x^{T} \Sigma_{\varepsilon} x / x^{T}\left(\Sigma_{\varepsilon}+\theta^{2} \Sigma_{\eta} \Sigma_{w} \Sigma_{\eta}\right) x$ exceeds one-half. But this is, by Rayleigh's principle (see Strang [25] pp. 253-254 and the proof of Theorem 5.2 below), ecquivalent to $U=\Sigma_{\varepsilon}\left(\Sigma_{\varepsilon}+\theta^{2} \Sigma_{\eta} \Sigma_{W} \Sigma_{\eta}\right)^{-1}$ having an eigenvalue greater than one-half, which is the relevant condition expressed in Theorem 4.2.

Under what conditions will $U$ have an eigenvalue exceeding one-half? The first is when $\Sigma_{w}$ is nearly singular. In this case, there is a mutual fund consisting of the insider's untraded securities whose distribution is nearly degenerate. The outsiders can then obtain a very reliable estimate of the insider's private information about the future value of the same mutual fund of tradable securities. But if all agents have the same information about the fund's future value, then all will wish to take the same side of any trade. Thus, to obtain an equilibrium in securities $\{1, \ldots, n\}, \Sigma_{w}$ must be some distance from a singular matrix.

There are two other occasions when $U$ has an eigenvalue exceeding one-half. First, when the insider is nearly risk neutral, and second when there is relatively little economy-wide uncertainty about the future value of the securities. When the insider is nearly risk neutral, he has little incentive to consider hedging tactics. Consequently, his motive for trade is almost entirely informational. Alternatively, when there is enough correlation between the future value of securities in that component representing economywide uncertainty (i.e. when $\Sigma_{\eta}$ is nearly singular), the insider can put together a mutual fund of tradable 
securities whose future value is almost certain. Thus, his demand for this mutual fund will be largely based on his private information, since the same mutual fund consisting of his untradable securities, $W_{1}, \ldots, W_{n}$, then also has an almost certain future value.

\subsection{EXISTENCE OF NONLINEAR EQUILIBRIA}

This section establishes that precisely the same condition characterizing the existence of a linear equilibrium characterizes the existence of any equilibrium at all in securities $\{1,2, \ldots, n\}$. Those readers primarily interested in applications of the eigenvalue condition for destructive interference may wish to skip this section and proceed immediately to Theorem 4.5 below.

Throughout this section, we maintain the hypothesis that the price schedule $\mathbf{P}(\cdot)$ is part of an equilibrium in securities $\{1,2, \ldots, n\}$. We begin with some notation. Given the equilibrium price schedule, $P(\cdot)$, define $F(S)=(S-\bar{S})^{T} P(S)+.5 \theta S^{T} \Sigma_{\eta} S$ for every $S$. Consequently, insider-type $\tau$ has an equilibrium utility of

$$
V(\tau)=\max _{S \in \mathbb{R}^{n}} S^{T} \tau-F(S) .{ }^{12}
$$

In this form, $\mathrm{V}(\cdot)$ is recognized as the conjugate of $F(\cdot) .^{13}$ As will be seen, the fact that one can express the insider's utility in this form, allows a good deal of convex analysis to be fruitfully applied. Indeed, it is helpful to introduce as well the conjugate of $\mathrm{V}(\cdot)$, namely

$$
f(S)=\max _{\tau \in \mathbf{R}^{n}} S^{T} \tau-V(\tau)
$$

for all $S$.

Thus, $f(\cdot)$ is the conjugate of the conjugate of $F(\cdot)$. Geometrically, $f(\cdot)$ is the greatest, in terms of pointwise values, convex function below $\mathrm{F}(\cdot)$ (see fact (b) in Appendix B). ${ }^{14}$ Consequently, one can equivalently define $V(\cdot)$ by replacing $F(\cdot)$ in its original definition above by $f(\cdot)$ (see fact (c) in 
Appendix B). That is,

$V(\tau)=\max _{i S} S^{T} \tau-F(S)=\max _{S} S^{T} \tau-f(S)$

for every $\tau$. However, the maximizers of the two problems need not be the same. With this in mind, let $M(\tau)=\left\{S \mid S^{T} \tau-F(S)=V(\tau)\right\}$ and $m(\tau)=\left\{S \mid S^{T} \tau-f(S)=V(\tau)\right\}$, and note that both sets of maximizers are nonempty for every $\tau$, since $P(\cdot)$ is an equilibrium price schedule.

For $g: \mathbf{R}^{\mathrm{n}} \rightarrow \mathbf{R}$, a convex function, let $\partial \mathrm{g}(\mathrm{x})$ denote the nonempty, compact, and convex set of subgradients of $\mathbf{g}$ at $\mathbf{x}$. That is

$\partial g(x)=\left\{q \in \mathbf{R}^{n} \mid g(y) \geq g(x)+q{ }^{T}(y-x)\right.$, for all $\left.y \in \mathbf{R}^{n}\right\}$

When $\partial g(x)=\{q\}$ is a singleton, we will sometimes simply write $\partial g(x)=q$. If $g$ is differentiable at $x$ we will denote its derivative there by $\nabla g(x)$. Of course $\partial g(x)$ is a singleton if and only if $\partial g(x)=\nabla g(x)$ (see Rockafellar [24], Theorem 25.1). Finally, for any convex subset $\mathbf{A}$ of $\mathbf{R}^{\mathbf{n}}$, denote by riA, the relative interior of $\mathbf{A}$.

Recall that $\Im(P)$ denotes the support of the outsiders' beliefs about the insider's type conditional on having observed the price vector $P$. Since the equilibrium price schedule $\mathbf{P}(\cdot)$ must be one to one, observing the price is equivalent to observing the insider's demand S. For simplicity only, we shall proceed from now on as if the outsiders actually observe the insider's demand S, rather than only the resulting price $\mathrm{P}$. Again, in any equilibrium, this is without any loss. Consequently, $\mathfrak{S}(\mathrm{S})$ shall denote the support of the outsiders' beliefs about the insider's type conditional upon having observed the insider's demand $\mathbf{S}$.

The strength of the D1 criterion lies in the restrictions it places upon the outsiders' beliefs about the insider's type subsequent to an out-of-equilibrium demand. Of course, Bayes' rule is employed subsequent to equilibrium demands. The following theorem establishes that in equilibrium Bayes' rule 
correctly restricts the outsiders' beliefs about the insider's type to the subgradient of $f(\cdot)$ evaluated at that insider-type's equilibrium demand. Moreover, it establishes that the D1 criterion leads to the same restriction on beliefs subsequent to out-of-equilibrium demands. It should be noted that this is the only use that we shall make of the criterion.

Proposition 4.3: In any equilibrium, $\mathfrak{I}(S) \subseteq \partial f(S)$ for all $S$.

Proof: Fix an equilibrium. Insider-type $\tau$ chooses $S$ to maximize $S^{T} \tau-F(S)$. Consequently, if $S_{0}$ is an equilibrium demand made by insider-type $\tau_{0}$, then $S_{0} \in M\left(\tau_{0}\right)$. But together, facts (c)(i) and (d)(ii) in Appendix B then imply that $\tau_{0} \in \partial f\left(S_{0}\right)$. Thus, the only insider-types who in equilibrium demand $S_{0}$ are members of $\partial \mathrm{f}\left(\mathrm{S}_{0}\right)$.

Next, consider a demand $S_{0}$ that is not made by any insider-type in equilibrium. In addition, suppose that there is a potential price response making some insider-type $\tau \notin \partial f\left(S_{0}\right)$ at least as well off as his equilibrium demand would have. We claim then that the same price response makes every insider-type in $\partial \mathrm{f}\left(\mathrm{S}_{0}\right)$ strictly better off. If this is true, then $\partial \mathrm{f}\left(\mathrm{S}_{0}\right)$ constitutes a prime suspect subset for $\mathrm{S}_{0}$, and therefore the D1 criterion implies that the outsiders' beliefs about the type of insider who demanded $S_{0}$ must have support in $\partial \mathrm{f}\left(\mathrm{S}_{0}\right)$.

To prove the claim, we need show that if for some $\tau \notin \partial f\left(S_{0}\right)$ and some price vector $P_{0}$ we have $S_{0}^{T} \tau-\left(S_{0}-\bar{S}\right)^{T} P_{0}-.5 \theta S_{0}^{T} \Sigma S_{0} \geq V(\tau)$, then we also have $S_{0}^{T} \tau_{0}-\left(S_{0}-\bar{S}\right)^{T} P_{0}-.5 \theta S_{0}^{T} \Sigma S_{0}>V\left(\tau_{0}\right)$ for every $\tau_{0} \in \partial f\left(S_{0}\right)$. But this is equivalent to showing that for any $\tau \notin \partial f\left(S_{0}\right)$, if $S_{0}^{T} \tau-y \geq V(\tau)$ then $s_{0}^{T} \tau_{0}-y>V\left(\tau_{0}\right)$ for all $\tau_{0} \in \partial f\left(S_{0}\right)$. To see that this is indeed the case, note that by fact (d)(ii) of Appendix $B, V(\tau)>S_{0}^{T} \tau-f\left(S_{0}\right)$ for $\tau \notin \partial f\left(S_{0}\right)$ and $V\left(\tau_{0}\right)=S_{0}^{T} \tau_{0}-f\left(S_{0}\right)$ for $\tau_{0} \in \partial f\left(S_{0}\right)$. Consequently, if $S_{0}^{T} \tau-y \geq V(\tau)$, then $y<f\left(S_{0}\right)$ so that $S_{0}^{T} \tau_{0}-y>V\left(\tau_{0}\right)$. QED 
Remark: Since $\mathrm{f}(\cdot)$ is, by fact (b) of Appendix B, convex, it is differentiable almost everywhere (Rockafellar [24] Theorem 25.5). Consequently, Proposition 4.3 implies that conditional on almost every demand, $\$$, of the insider, the outsiders' beliefs are a point mass on $\mathrm{f}(\mathbf{S})$.

Despite Proposition 4.3, we still may know very little about the outsiders' beliefs conditional on equilibrium demands made by the insider. For instance, in the one security case, if $f(\cdot)$ fails to be differentiable at $S_{0}$, then $S_{0}$ is chosen by an open set of insider-types. Indeed, if $f(S)$ is piecewise linear yet everywhere above the function $g(S)=S^{2}$, say, then $f$ has infinitely many kinks and almost every insidertype maximizes uniquely by choosing an amount of the single security at which $f$ is kinked. Consequently, almost all of the equilibrium action might involve points at which $\mathrm{f}$ fails to be differentiable whereas Proposition 4.3 provides detailed information only when $\mathrm{f}$ is differentiable.

It is therefore necessary to further investigate the nature of the insider's equilibrium demands, especially those that might be associated with kinks (non-differentiabilities) in $f(\cdot)$. With this in mind, call a demand $S$ by the insider a peak if for some insider-type, $\tau, m(\tau)=\{S\}$. Consequently, if $S$ is a peak, then $S$ is the unique maximizing demand for some insider-type. ${ }^{15}$

A.s it turns out, in any equilibrium, every $S$ is a peak. ${ }^{16}$ Consequently, every $S$ is demanded by some insider-type; there are no out-of-equilibrium demands. This is stated as part of the following theorem.

Proposition 4.4: In any equilibrium every $S$ is a peak, and $F(\cdot$ is convex and equal to $f(\%$ Consequently, for almost every $S,(i)$ the outsiders' beliefs are a point mass on $\nabla F(S)$, and (ii) $F(\cdot)$ satisfies the following differential equation:

$$
F(S)=\nabla F(S)^{T} U(S-\bar{S})-\phi\left[S^{T} \Sigma_{0}(\bar{S}+1)-\bar{S}^{T} \Sigma_{0} 1\right]+S^{T}\left[\phi \Sigma_{0}+.5 \theta \Sigma_{\eta}\right] S
$$

Proof: See Appendix A. 
To understand Proposition 4.4, it is enough to consider the single security case. First, to see that $F(\cdot)$ must be convex, suppose, as depicted in Figure 1, that it is not. Before continuing, note the geometry of the figure: If insider-type $\imath$ chooses $S$ to maximize $S \tau-F(S)$, then at the maximizing demand, $\hat{\mathbf{S}}$, it must be the case that $\hat{\imath} \in \partial \mathrm{F}(\hat{\mathbf{S}})$. I.e., the line with slope $\hat{\imath}$ through the point $(\hat{\mathbf{S}}, \mathrm{F}(\hat{\mathbf{S}}))$ must be tangent to $F\left({ }^{\circ}\right)$ at $\hat{S}$ (see the figure). With this in mind, insider-type $\tau=0$ will, according to Figure 1, choose either $S_{0}$ or $S_{1}$. Moreover, no insider-type chooses $S \in\left(S_{0}, S_{1}\right)$.

[FIGURES 1 AND 2 HERE.]

Thus, every demand in $\left(S_{0}, S_{1}\right)$ is an out-of-equilibrium demand and the D1-criterion restricts the outsiders' beliefs subsequent to any such demand. Indeed, according to Proposition 4.3 , if $\tilde{S} \in\left(S_{0}, S_{1}\right)$ then the support of the outsiders' beliefs about the insider's type must be contained in $\partial \mathrm{f}(\tilde{\mathrm{S}})$. But Figure 2 shows that $\partial f(\tilde{S})=\{0\}$. I.e., $f(\cdot)$ is differentiable on $\left(S_{0}, S_{1}\right)$ with derivative zero there. Consequently if any demand $\tilde{S} \in\left(S_{0}, S_{1}\right)$ is made, the outsiders will believe with certainty that the insider is type $\tau=$ 0. Hence, each outsider's first-order condition (they choose $S^{\alpha}$ ) subsequent to such a demand is $U \cdot 0-P(\tilde{S})-\phi \Sigma_{0} S^{\alpha}=0$, which implies, since $\int_{0}^{1} S^{\alpha} d v=1-\tilde{S}$ in equilibrium, that $P(\tilde{S})=\phi \Sigma_{0}(\tilde{S}-1)$ for all $\tilde{S} \in\left(S_{0}, S_{1}\right)$.

But this leads to a contradiction. For if $P(S)=\phi \Sigma_{0}(S-1)$ on $\left(S_{0}, S_{1}\right)$, then $F(S)=(S-\bar{S})^{T} \mathbf{P}(S)+.5 \theta S^{T} \Sigma_{\eta} S$ is strictly convex on $\left(S_{0}, S_{1}\right)$ which, according to Figure 1, it is not. Note that we've actually established that $F(\cdot)$ must be strictly convex, so that every $S$ is a peak. It also follows that $F(\cdot)=f(\cdot)$ since the greatest convex function below $F(\cdot)$ (now known to be convex) 
is $F(\cdot)$ itself.

Finally, that $F(\cdot)$ satisfies the stated differential equation can be derived from equilibrium considerations as follows. Together, Proposition 4.3; $f(\cdot)=F(\cdot)$; and the almost everywhere differentiability of convex functions, imply that for almost every $S$, the outsiders' beliefs must be a point mass on $\nabla F(S)$. Each outsider's first-order condition is then $U \nabla F(S)-P(S)-\phi \Sigma_{0} S^{\alpha}=0$, for almost every $S$, the insider's demand, and where $S^{\alpha}$ is outsider $\alpha$ 's subsequent demand. Since, in equilibrium, $\int_{0}^{1} S^{\alpha} d v=1-S$ this implies $P(S)=U \nabla F(S)-\phi \Sigma_{0}(1-S)$, for almost every $S$. The differential equation results by substitution into $F(S)=(S-\bar{S})^{T} P(S)+.5 \theta S^{T} \Sigma_{\eta} S$, the definition of $F(\cdot)$.

In order to show that the eigenvalue condition characterizes the existence of an equilibrium in securities $(1,2, \ldots, n)$, a final step remains. Recall that because we are in equilibrium,

$$
V(\tau)=\max _{S} S^{T} \tau-F(S)
$$

must be well-defined for every insider-type $\tau$. Clearly, a necessary condition for this is that:

$$
\mathbf{F}(\mathbf{S}) \rightarrow+\infty \text { as }\|\mathbf{S}\| \rightarrow+\infty \text {. }
$$

For if $\left\{S^{n}\right\}_{i=1}^{\infty}$ violates (16) then the insider's maximization problem has no solution for insider-type $\tau^{*}$, an appropriate limit point of $\left\{S^{n} / \| S^{n} \mid\right\}_{n=1}^{\infty}$. The final step shows that if an equilibrium exists, so that in particular (16) must be satisfied, then in order to satisfy (16), every eigenvalue of $U$ must be less than onehalf.

So suppose that there is an equilibrium in securities $\{1,2, \ldots, n\}$. By Proposition 4.4,

$$
F(S)=F(S)^{T} U(S-\bar{S})-\phi\left[S^{T} \Sigma_{0}(\bar{S}+1)-\bar{S}^{T} \Sigma_{0} 1\right]+S^{T}\left[\phi \Sigma_{0}+.5 \theta \Sigma_{\eta}\right] S,
$$


for almost every S.

By Lemmas A.1 and A.2 of Appendix A, $U$ can be written as $U=\Gamma^{-1} \Lambda \Gamma$, where $\Lambda$ is a diagonal matrix, whose diagonal elements consist of the eigenvalues, $\lambda_{i} \in(0,1)$, of $U$. Let $x=\Gamma(S-\bar{S})$, $A=\left(\Gamma^{-1}\right)^{T}\left[\phi \Sigma_{0}+.5 \theta \Sigma_{\eta}\right] \Gamma^{-1}, b=\Gamma^{-1}\left[\phi \Sigma_{0}(\bar{S}-1)+\theta \Sigma_{\eta} \bar{S}\right]$, and $d=.5 \theta \bar{S}^{T} \Sigma_{\eta} \bar{S}$. Also, define H:R $\rightarrow \mathbf{R}$ by $H(x)=F\left(\Gamma^{-1} x+\bar{S}\right)$. Hence, (17) becomes:

$$
H(x)=\nabla H(x)^{T} \Lambda x+b^{T} x+x^{T} A x+d
$$

for almost every $x$. Note that $H$ inherits, from $F$, convexity.

Next define for each $x, g:(0, \infty) \rightarrow R$ by $g(t)=H\left(x_{1} t^{\lambda_{1}}, \ldots, x_{n} t t_{n}\right)$. Because $H$ is convex, it is also differentiable for almost every $x$. Therefore, for almost every $x$ with $\|x\|=1$ ( $n-1$ dimensional Lebesgue measure), $\mathrm{g}$ is differentiable for almost every $\downarrow 0$. Fix then $\mathrm{x}^{*}$ such that $\left\|\mathrm{x}^{*}\right\|=1$, no component of $\mathrm{x}^{*}$ is zero, and $g(t)=h\left(x_{1}^{*} t^{\lambda_{1}}, \ldots, x_{n}^{*} t \lambda_{n}\right)$ is differentiable for almost every $\triangleright 0$. Consequently, by (18)

$$
\operatorname{tg}^{\prime}(t)=g(t)-\sum_{i=1}^{n} b_{i} x_{i}^{*} t^{\lambda_{i}}-\sum_{i j} x_{i}^{*} a_{i j} x_{j}^{*} t^{\lambda_{i}+\lambda_{j}}-d
$$

for almost every $>0$. Now since $H$ is convex, it is Lipshitz. Therefore, for every $\alpha>0, g$ is Lipshitz on $[\alpha, \infty)$ and it can therefore be recovered from its derivative (where defined). Hence we may solve the differential equation (19), to conclude that $g$ must be of the form 


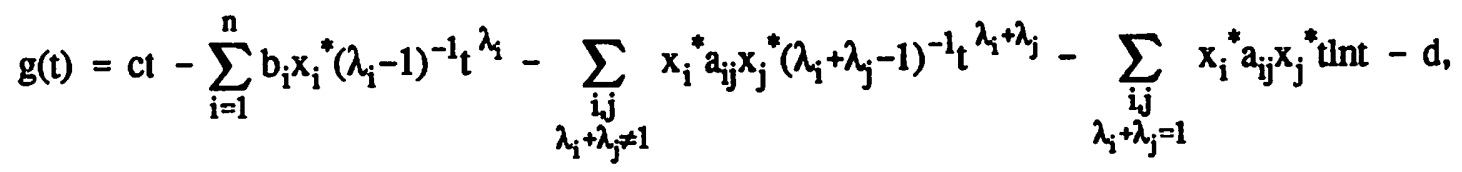

for $\gg 0$, and some constant $c$.

Now let $\lambda_{m}$ denote the largest eigenvalue of $U$. If $\lambda_{m}=1 / 2$, then from (20) the leading term of $g(t)$ is

$$
-\left\{\sum_{\substack{i: \lambda_{i}=.5 \\ j: \lambda_{j}=.5}} x_{i}^{*} a_{i j} x_{j}^{*}\right\}^{t h t}
$$

whereas if $\lambda_{\mathrm{m}}>1 / 2$ the leading term is

$$
-\left\{\sum_{\substack{i: \lambda_{i}=\lambda_{m} \\ j: \lambda_{j}=\lambda_{m}}} x_{i}^{*} a_{i j} x_{j}^{*}\right\}\left(2 \lambda_{m}-1\right)^{-1} t^{2 \lambda_{m}}
$$

In either case because the terms in curly brackets are strictly positive (recall that $A$ is positive definite and no component of $x^{*}$ is zero), we have $g(t) \rightarrow-\infty$ as $t \rightarrow \infty$. But this means that $F\left(\Gamma^{-1}\left(x_{1}^{*} t^{\lambda_{1}}, \ldots, x_{n}^{*} t \lambda_{n}\right)^{T}+\bar{S}\right) \rightarrow$ $-\infty$ as $\mathrm{t} \rightarrow \infty$, contradicting (16).

Consequently, a necessary condition for the existence of an equilibrium (linear or otherwise) in securities $\{1,2, \ldots, n\}$ is that every eigenvalue of $U$ must be less than one-half. Combined with Theorem 4.2 we ottain the following characterization theorem. 
Theorem 4.5: The following statements hold except possibly for a closed subset of parameter values having Lebesgue measure zero: There is an equilibrium in securities $\{1,2, \ldots, n\}$ if and only if every eigenvalue of the outsiders' update matrix, $U$, is less than one-half. Furthermore, this is precisely when a linear equilibrium in these securities exists as well.

\section{THE GENERAL CASE}

Now that most of the work is done, we remind the reader that our broader notion of an equilibrium includes not only the agents' equilibrium demands, and the equilibrium price schedule, but also the collection of tradable securities. As we are now aware, under certain conditions there can be no equilibrium in which all $\mathrm{n}$ securities are tradable. So, viewing the collection of tradable securities as something that is determined in equilibrium, which has been our point of view from the beginning, it is natural to now fix the market structure so that only a strict subset, say $\{1, \ldots, k\}$, of the set of $n$ potentially tradable securities is tradable. Note that one cannot simply replace the $\mathbf{n}$ in the previous section with $k$, because there are now additional untradable securities (namely, securities $k+1, \ldots, n$ ) that must simply be held by all agents endowed with them. Moreover, the insider has private information about the future values of these untradable securities, values which might be correlated with those of the tradable securities. Despite these differences, the analysis of this case proceeds along lines that are very similar to those in the previous two sections. Consequently, we provide only the results.

$$
\text { Let } \left.\mathrm{U}_{k}=\mathrm{J}_{k}^{\mathrm{T}} \Sigma_{\varepsilon} \mathrm{J}_{k}\left[\mathrm{~J}_{k}^{\mathrm{T}}\left(\theta^{2} \Sigma_{\eta} \Sigma_{w} \Sigma_{\eta}+\Sigma_{\varepsilon}\right) \mathrm{J}_{k}\right]^{-1} \text { where } \mathrm{J}_{k}^{\mathrm{T}} \text { (the transpose of } \mathrm{J}_{k}\right) \text { is the kxn matrix }\left(\mathrm{I}_{k} 0_{\mathrm{kx}(n-k)}\right) \text {, }
$$

$I_{k}$ is the $k \times k$ identity matrix, and $0_{k x(n-k)}$ is a $k \times(n-k)$ matrix of zeros. An equilibrium in securities $\{1,2, \ldots, \mathrm{k}\}$ is defined analogously to an equilibrium in securities $\{1,2, \ldots, \mathrm{n}\}$ except that, in addition, each agent must retain his endowed amounts of securities $k+1$ through $\mathbf{n}$.

Theorem 5.1: The following statements hold except possibly for a closed set of parameter values having Lebesgue measure zero. There is an equilibrium in securities $\{1,2, \ldots, k\}$ if and only if every eigenvalue 
$\lambda_{1}, \ldots, \lambda_{k}$ of $U_{k}$ is less than 112. Furthermore, this is precisely when a linear equilibrium in securities $\{1,2, \ldots, k\}$ exists as well.

Note that by simply renumbering the securities, Theorem 5.1 provides a necessary and sufficient condition for the existence of an equilibrium in any subset of the $\mathbf{n}$ potentially tradable securities. Consequently, we are now able to determine which security markets can open together in equilibrium. We shall say that a subset of the set of securities constitutes an equilibrium market structure (or is consistent with equilibrium) if there is an equilibrium in the given subset of securities.

The theorem to follow indicates that if destructive interference occurs, then adding still more securities to the market cannot result in an equilibrium market structure either. For the remainder of this section we will assume that the vector of parameter values $\left(\phi, \theta, \Sigma_{\eta}, \Sigma_{w}, \Sigma_{\varepsilon}\right)$ is not a member of the exceptional set described in Theorem 5.1.

Theorem 5.2: If securities 1 through $k$ do not constitute an equilibrium market structure, then neither do securities 1 through $k+1$.

Proof: In view of Theorem 5.1, it suffices to show that the largest eigenvalue of $U_{k+1}$ is at least as large as the largest eigenvalue of $U_{k}$. We recall Rayleigh's principle (see Strang [25], pp. 253-254) which states that if $A$ is a symmetric nxn matrix, then the largest eigenvalue of $A$ is given by:

$$
\max _{x \in \mathbb{R}^{n}} \frac{x^{\top} A x}{x^{T} x}
$$

Now, if $A$ and $B$ are symmetric nxn positive definite matrices, then the eigenvalues of $A(A+B)^{-1}$ coincide with those of $Q^{T} A Q$, where the positive definite matrix $A+B$ is written as $\left(Q^{-1}\right)^{T}\left(Q^{-1}\right)$ for some nonsingular matrix $Q$. Thus, the largest eigenvalue of $A(A+B)^{-1}$, being also the largest eigenvalue of 
$Q^{T} A Q$ is, by Rayleigh's principle:

$$
\max _{\substack{x \in R^{n} \\ x \neq 0}} \frac{x^{T} Q^{T} A Q x}{x^{T} x}=\max _{\substack{y \in R^{n} \\ y \neq 0}} \frac{y^{T} A y}{y^{T}\left(W^{-1}\right)^{T}\left(W^{-1}\right) y,}
$$

where the equality follows from the change of variable $y=W x$. Hence, the largest eigenvalue of $A(A+B)^{-1}$ is given by

$$
\max _{\substack{y \in \mathbb{R}^{n} \\ y \neq 0}} \frac{y^{T} A y}{y^{T}(A+B) y}
$$

So, letting $\pi_{k}$ denote the largest eigenvalue of $U_{k}$ yields:

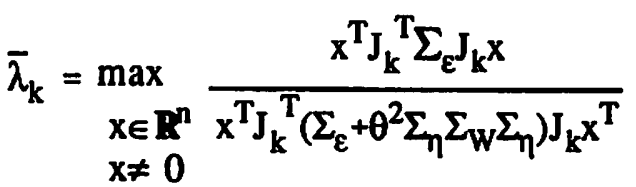

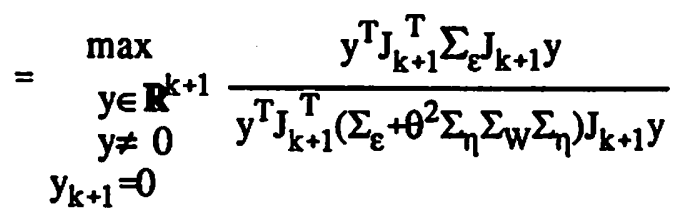

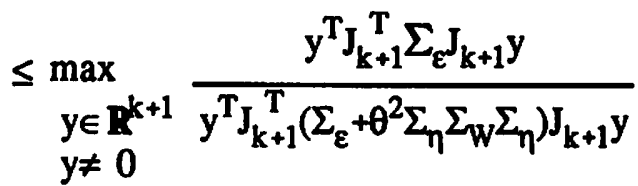

$$
\begin{aligned}
& =\bar{\lambda}_{k+1} \text {. }
\end{aligned}
$$

That is, the largest eigenvalue of $U_{k}$ is nondecreasing in $k$. Q.E.D.

Theorem 5.2 conforms nicely with our earlier intuition for the existence of an equilibrium. For suppose there is no equilibrium in securities 1 through $k$. Then there must be a mutual fund made up of these securities for which the insider's trading motive is relatively informational. Since adding a security 
does not preclude one from constructing this same mutual fund, adding a security can not create an equilibrium where there was none before.

We now provide a condition under which no nonempty subset of the set of securities is consistent with equilibrium. Thus, the only equilibrium is the trivial no trade equilibrium in which no securities are tradable. The following is immediate from Theorem 5.2:

Corollary 5.3: If no single security is consistent with equilibrium, then no nonempty collection of security markets is consistent with equilibrium. Moreover, this is the case precisely when $\sigma_{\varepsilon_{i}}^{2}\left[\theta^{2} \sigma_{\eta_{i}}^{4} \sigma_{w_{i}}^{2}+\sigma_{\varepsilon_{i}}^{2}\right] \frac{1}{2}$ for every $i=1,2, \ldots, n$.

Corollary 5.3 provides a generalization of the market breakdown condition in Bhattacharya and Spiegel [6]. Roughly speaking, when at least half of the insider's trading activity in every security can be attributed to his private information, no market nor any subset of markets can open. Next, we provide a condition under which new securities can be added to the market so that the resulting market structure is consistent with an equilibrium.

For any subset $M$ of $\{1,2, \ldots, n\}$, any vector $x$, and any nxn matrix $A$, let $x_{M}=\left(x_{j}\right)_{i \varepsilon M}$, and let $A^{M}$ be the $|M||\times| M \mid$ matrix with $i-j{ }^{\text {th }}$ element $a_{i j}$ for $i, j \in M$. Also, denote by $U_{M}$ the analogue of $U_{k}$ where the labels $\{1,2, \ldots, \mathrm{k}\}$ are replaced by those in $\mathrm{M}$.

Corollary 5.4: Let $M$ and $N$ be two disjoint subsets of the set of securities $\{1,2, \ldots, n\}$, and suppose that $\varepsilon_{M}$ and $\varepsilon_{N} ;$ as well as $\eta_{M}$ and $\eta_{N}$ and $W_{M}$ and $W_{N}$ are independent. If both subsets of securities $M$ and $N$ are consistent with equilibrium, then so is $M \cup N$.

Proof: Given the above independence assumptions, 


$$
\mathrm{U}_{\mathrm{MUN}}=\left[\begin{array}{cc}
\Sigma_{\varepsilon}^{\mathrm{M}} & 0 \\
0 & \Sigma_{\varepsilon}^{\mathrm{N}}
\end{array}\right]\left[\begin{array}{cc}
\Sigma_{\varepsilon}^{\mathrm{M}}+\theta^{2} \Sigma_{\eta}^{\mathrm{M}} \Sigma_{w}^{\mathrm{M}} \Sigma_{\eta}^{\mathrm{M}} & 0 \\
0 & \Sigma_{\varepsilon}^{\mathrm{N}}+\theta^{2} \Sigma_{\eta}^{\mathrm{N}} \Sigma_{w}^{N} \Sigma_{\eta}^{N}
\end{array}\right]^{-1}
$$

and so the eigenvalues of $U_{M U N}$ are simply the union of the eigenvalues of $U_{M}$ and $U_{N}$. Therefore each of these eigenvalues is less than $1 / 2$. The result now follows from Theorem 5.1. Q.E.D.

Thus, introducing new securities into the market whose future values are independent of those of the current collection of tradable securities does not result in destructive interference. The next corollary turns to the opposite extreme and shows that sufficient correlation between a collection of securities' future values precludes the existence of an equilibrium in this collection. Indeed, it shows somewhat more.

Corollary 5.5: If $J_{k}^{T}\left(\theta^{2} \Sigma_{\eta} \Sigma_{w} \Sigma_{\eta}\right) J_{k}$ is close enough to a singular matrix, then securities $\{1,2, \ldots, k\}$ do not constitute an equilibrium market structure.

Proof: Let $\left\{A_{m}\right\}$ be a sequence of $n \times n$ symmetric, positive definite matrices converging to $A$, where $J_{k}^{T} A J_{k}$ is singular. Let $\Lambda_{m}$ denote the kxk diagonal matrix of eigenvalues of $J_{k}^{T} \Sigma_{\varepsilon} J_{k}\left[\left(J_{k}^{T}\left(A_{m}+\Sigma_{\varepsilon}\right) J_{k}\right)\right]^{-1}$, a kxk diagonalizable matrix (see Lemma A.1 of Appendix A). Since the eigenvalues of a matrix are continuous in its entries (see, for instance, Horn and Johnson [16], p. 539), $\Lambda_{\mathrm{m}}$ converges to $\Lambda$ the kxk diagonal matrix of eigenvalues of $\mathrm{J}_{k}^{T} \Sigma_{\varepsilon} J_{k}\left[\left(J_{k}^{T}\left(A+\Sigma_{\varepsilon}\right) J_{k}\right)\right]^{-1}$, which has an eigenvalue greater than $1 / 2$ since its inverse $\left[\mathrm{J}_{k}^{\mathrm{T}} \mathrm{AJ} \mathrm{J}_{k}\right]\left[\mathrm{J}_{k}^{\mathrm{T}} \Sigma_{\varepsilon} \mathrm{J}_{k}\right]^{-1}+\mathrm{I}_{k}$ has an eigenvalue of 1 . Hence, for $m$ large enough, $J_{k}^{T} \Sigma_{\varepsilon} J_{k}\left[\left(J_{k}^{T}\left(A_{m}+\Sigma_{\varepsilon}\right) J_{k}\right)\right]^{-1}$ has an eigenvalue larger than $1 / 2$. Therefore, if $J_{k}^{T}\left(\theta^{2} \Sigma_{\eta} \Sigma_{w} \Sigma_{\eta}\right) J_{k}$ is close enough to a singular matrix, then $U_{k}$ has an eigenvalue exceeding $1 / 2$. Now, apply Theorem 5.1. Q.E.D.

One interpretation of the above result is that futures or options markets can potentially eliminate 
the markets in the underlying securities. To see this, allow $\varepsilon$ to represent near term events about which the insider has private information. To the extent that there are some near term events that have a differential impact upon the future and the stock retum (for example, a large eamings announcement that results in a one time dividend bonus) the matrix $\Sigma_{\varepsilon}$ will likely be well conditioned. Allow $\eta$ to represent long term events about which no one knows a great deal. Now, long term events that are realized after trade may' well affect both the stock price and its corresponding futures price about equally. Consequently, $\Sigma_{\eta}$ may be nearly singular. If $\Sigma_{\eta}$ is near enough to a singular matrix, Corollary 5.5 implies that there can be no equilibrium in which the futures market is also present. Thus, introducing the futures market may induce a persistent inability of the market-maker in the underlying securities to clear the market. Consequently, the present model provides a mechanism through which the introduction of a futures market may cause the market in the underlying securities to break down. In our terminology, futures markets can destructively interfere with markets in the underlying securities.

Nimerical examples of destructive interference abound. We now provide one. Consider an economy with only two potentially tradeable securities $(n=2)$ and covariance matrices:

$\Sigma_{\eta}=\left[\begin{array}{cc}1.0 & .6 \\ .6 & 1.0\end{array}\right] \Sigma_{W}=\left[\begin{array}{cc}100 & 0 \\ 0 & 100\end{array}\right] \Sigma_{\varepsilon}=\left[\begin{array}{cc}20 & 0 \\ 0 & 20\end{array}\right]$

If either security is the only one that is tradeable, the largest (and only) eigenvalue of the insider's update matrix is .128. Since this is less than one-half, there is an equilibrium in which security 1 trades alone and an equilibrium in which security 2 trades alone. However, if both securities are tradable, the largest eigenvalue of the insider's update matrix is .556 . Hence, by Theorem 4.5 , there is no equilibrium in which both securities 1 and 2 are tradable. In particular then, if security 1 is currently trading, the introduction of security 2 may result in the elimination of security 1 .

A question that arises in any multi-security economy is whether Giffen securities (that is, a 
security whose demand increases with its price) can arise. As in Admati [2], we find that they can. In the context of our model a security is Giffen if the outsiders' aggregate demand for it is increasing in its price. This is the natural definition since the outsiders are price-takers. But since, in equilibrium, the outsiders' aggregate excess demands must equal the insider's excess supply, it suffices to find a security such that the insider's demand for it is negatively related to its price. The following example illustrates this possibility.

Giffen Security Example: Suppose that there are only two securities and that they both trade. Let $\Sigma_{\varepsilon}=\left[\begin{array}{cc}40 & 19 \\ 19 & 10\end{array}\right] \Sigma_{\eta}=\left[\begin{array}{cc}11 & 22 \\ 22 & 70\end{array}\right] \Sigma_{w}=\left[\begin{array}{cc}6 & 20 \\ 20 & 100\end{array}\right], \theta=2$ and $\phi=.0001$. Consequently, the outsiders' update matrix, $\mathrm{U}$, has a largest eigenvalue slightly less than .2 , so that a linear equilibrium exists. Solving (13) for the linear equilibrium produces,

$$
\left(\frac{\partial P}{\partial S}\right)^{-1}=\left[\begin{array}{cc}
-.05 & 1.74 \\
-75.4 & 161.74
\end{array}\right]
$$

Thus, security one is Giffen.

\section{CONCLUSION}

The model presented in this paper facilitates the study of a phenomenon particular to imperfectly competitive multi-security markets: destructive interference. In contrast to competitive settings, adding securities to a system here does not always foster additional trade. A new security may destructively interfere with one or more securities already present. It is therefore possible, for instance, that the birth of a futures market might eliminate the market in the underlying securities. Or, a firm may ultimately be unable to issue a new security if it has already issued a similar security in the past; it may be able to issue one combined security for all its divisions, but not separate securities for its different divisions. 
The possibility of destructive interference suggests that equilibrium in the presence of insider trading is a delicate matter. The nature of the equilibrium collection of trading securities can be dramatically altered by the addition of a single new security. The extent to which these issues arise when there are many insiders, rather than just one, is a question which awaits future work. 


\section{APPENDIX A}

Lemma A.1: If $A$ and $B$ are real, symmetric nxn matrices and $B$ is positive definite, then $A B$ is diagonalizable, its eigenvalues and eigenvectors are real, and it has a real diagonalizing matrix.

Proof: Let $\lambda$ be an eigenvalue of $A B$ and $x$ a corresponding eigenvector. Then $A B x=\lambda x$. Since $B$ is real, symmetric and positive definite, $B=W^{T} W$ for some real nxn nonsingular matrix $W$ (see Strang [25], Theorem 6c, p. 241). So, letting $y=W x$ we have $W A W^{T} y=\lambda y$. But $W^{T} A W$ is then real and symmetric. Hence, $W^{T} A W$ has $n$ linearly independent (indeed, mutually orthogonal) real eigenvectors $y_{1}, \ldots, y_{n}$. corresponding to its $n$ (not necessarily distinct) real eigenvalues $\lambda_{1}, \ldots, \lambda_{n}$. But $\lambda_{1}, \ldots, \lambda_{n}$ are then the $n$ (real) cigenvalues of $A B$, and $x_{1}=W^{-1} y_{1}, \ldots, x_{n}=W^{-1} y_{n}$ are corresponding (real) eigenvectors. Since $W^{-1}$ is of full rank, $x_{1}, \ldots, x_{n}$ are linearly independent. Hence, the real $n x n$ nonsingular matrix whose $i^{\text {th }}$ column is $\mathbf{x}_{\mathbf{i}}$ diagonalizes AB. Q.E.D.

Lemma A.2: If $A$ and $B$ are $n x n$ symmetric positive definite matrices, then the eigenvalues of $A(A+B)^{-1}$ lie strictly between 0 and 1.

Proof: Let $\lambda$ be an eigenvalue of $A(A+B)^{-1}$ and $x$ be a corresponding eigenvector. Then $A(A+B)^{-1} x=\lambda x$, so letting $y=(A+B)^{-1} x$ yields $A y=\lambda(A+B) y$. But this means that $\lambda=y^{T} A y / y^{T}(A+B) y$ which is well defined (since $y \neq 0$ and $A$ and $B$ are positive definite) and lies strictly between zero and one. Q.E.D.

Given two $m \times n$ matrices $A$ and $B$, the Hadamard product of $A$ and $B$, denoted $A * B$ is the $m \times n$ matrix whose $\mathrm{i}-\mathrm{j}^{\text {th }}$ entry is $\mathrm{a}_{\mathrm{ij}} \mathrm{b}_{\mathrm{ij}}$ (that is, matrices are ${ }^{*}$-multiplied by multiplying corresponding entries. 
Lemma A.3: Suppose that $A$ and $B$ are $n \times n$, symmetric matrices and that $A$ is positive definite and $B$ is positive semidefinite. Then $A^{*} B$ is positive definite if and only if $B$ has no diagonal entry equal to zero. Proof: St:e Horn and Johnson [16], Theorem 5.2.1.

Lemma 4.1: Except possibly for a closed set of parameter values having Lebesgue measure zero, there is a solution, A, to (14) which is nonsingular and which renders both sides of (14) positive definite, if and only if every eigenvalue of $U$ is less than $1 / 2$.

Proof: We first show that (14) possesses a solution, A, satisfying the positive definiteness requirement if and only if every eigenvalue of $U$ is less than one-half. We then inquire as to when this solution is, in addition, nonsingular.

We begin by diagonalizing $\mathrm{U}$. That $\mathrm{U}$ can be diagonalized follows from its definition and Lemma A.1. Write $U$ as $\Gamma^{-1} \Lambda \Gamma$, where $\Lambda$ is an nxn diagonal matrix whose real diagonal entries are the eigenvalues $\lambda_{1}, \ldots, \lambda_{n}$ of $U$. By Lemma A.2, each $\lambda_{i}$ lies strictly between zero and one. Now let $B=\Gamma A \Gamma^{T}$, $\mathrm{E}=\phi \Gamma \Sigma_{0} \Gamma^{\mathrm{T}}$ and $\mathrm{F}=\theta \Gamma \Sigma_{\eta} \Gamma^{\mathrm{T}}$. Premultiplying (14) by $\Gamma$ and postmultiplying by $\Gamma^{\mathrm{T}}$ yields:

$$
B+B^{T}+F^{i}=\Lambda^{-1}(B-E)
$$

Let $\mathrm{H}$ deriote the matrix $\mathrm{B}+\mathrm{B}^{\mathrm{T}}+\mathrm{F}$ and $h_{\mathrm{ij}}$ its $\mathrm{i}-\mathrm{j}^{\text {th }}$ element. Since $\Gamma$ is of full rank it suffices to show that (A.1) has a solution, $B$, such that $H=B+B^{T}+F$ is rendered positive definite, if and only if each diagonal entry $\lambda_{i}$ of $\Lambda$ is less than one-half.

So, suppose that a solution, $B$, to (A.1) exists and that $\mathrm{H}$ is positive definite. We wish to show that each $\lambda_{i}<1 / 2$. Letting lower case letters represent elements of the corresponding matrices in (A.1) yields for every $i, j=1, \ldots, n$

$$
b_{i j}+b_{j i}+f_{i j}=\frac{1}{\lambda_{i}}\left(b_{i j}-e_{i j}\right)
$$

But then also, recalling that $E$ and $F$ are symmetric: 


$$
b_{j i}+b_{i j}+f_{i j}=\frac{1}{\lambda_{j}}\left(b_{j i}-e_{i j}\right) .
$$

Now, putting $\mathrm{i}=\mathrm{j}$ yields $\left(2 \lambda_{\mathrm{i}}-1\right) \mathrm{b}_{\mathrm{ii}}=-\left(\lambda_{\mathrm{i}} \mathrm{f}_{\mathrm{ii}}+\mathrm{e}_{\mathrm{ii}}\right)<0$, since $\mathrm{E}$ and $\mathrm{F}$ are positive definite, and $\lambda_{i} \in(0,1)$. Therefore $\lambda_{i} \neq 1 / 2$, and so

$$
b_{i i}=\left(1-2 \lambda_{i}\right)^{-1}\left(\lambda_{i j} f_{i i}+e_{i i}\right)
$$

Recalling that $\mathrm{H}=\mathrm{B}+\mathrm{B}^{\mathrm{T}}+\mathrm{F}$, we then have:

$$
h_{i i}=\left(1-2 \lambda_{i}\right)^{-1}\left(2 e_{i i}+f_{i j}\right) \text {. }
$$

Since the diagonal elements of $\mathrm{H}$, given by (A.5), must be positive if $\mathrm{H}$ is to be positive definite, each $\lambda_{i}$ must be less than $1 / 2$.

We now show the converse, namely, that if each $\lambda_{i}$ is less than $1 / 2$, then (A.1) possesses a solution, $B$, and it renders $H$ positive definite. Note first that when $\lambda_{i}<1 / 2$ for each $i,(A .2)$ and (A.3) together determine B. Indeed

$$
b_{i j}=\left(1-\lambda_{i}-\lambda_{j}\right)^{-1}\left[\left(1+\lambda_{i}-\lambda_{j}\right) e_{i j}+\lambda_{i} f_{i j}\right]
$$

Since $H=B+B^{T}+F$, we then have

$$
h_{i j}=\left(1-\lambda_{i}-\lambda_{j}\right)^{-1}\left(2 e_{i j}+f_{i j}\right) \text {. }
$$

In view of Lemma A.3, equation (A.7), and noting that $E$ and $F$ are positive definite, $H$ will be positive definite if the matrix whose $i-j$ th entry is $\left(1-\lambda_{i}-\lambda_{j}\right)^{-1}$, is positive semidefinite. (Note that no diagonal entry is zero.) That this is indeed the case when each $\lambda_{i} \in(0,1 / 2)$ is established by noting that: 


$$
\begin{aligned}
\left(1-\lambda_{i}-\lambda_{j}\right)^{-1} & =\left[\left(1-\lambda_{i}\right)\left(1-\lambda_{j}\right)-\lambda_{i} \lambda_{j}\right]^{-1} \\
& =\left[\left(1-\lambda_{i}\right)\left(1-\lambda_{j}\right)\right]^{-1}\left[1-\left[\frac{\lambda_{i}}{1-\lambda_{i}}\right]\left[\frac{\lambda_{j}}{1-\lambda_{j}}\right]\right]^{-1} \\
& =\left[\left(1-\lambda_{j}\right)\left(1-\lambda_{j}\right)\right]^{-1}\left[1+\left[\frac{\lambda_{i}}{1-\lambda_{i}}\right]\left[\frac{\lambda_{j}}{1-\lambda_{j}}\right]+\left[\frac{\lambda_{i}}{1-\lambda_{i}}\right]^{2}\left[\frac{\lambda_{j}}{1-\lambda_{j}}\right]^{2}+\ldots\right]
\end{aligned}
$$

where the infinite series converges since $\lambda_{i} \in(0,1 / 2)$ implies that $\lambda_{i} /\left(1-\lambda_{i}\right) \in(0,1)$. Hence, $\left(1-\lambda_{i}-\lambda_{j}\right)^{-1}$ is the (convergent) sum of terms of the form $\alpha_{j} \alpha_{j}$. But every nxn matrix having $i-j$ th entry $\alpha_{i} \alpha_{j}$ is positive semidefinite. Consequently, the matrix $\left(\left(1-\lambda_{i}-\lambda_{j}\right)^{-1}\right)$ is the (convergent) sum of positive semidefinite matrices and is therefore positive semidefinite.

We have therefore proven the following. If equation (14) possesses a solution, A, which renders both sides of (14) positive definite, then every eigenvalue of $U$ must be less than $1 / 2$. Hence, the same conclusion holds a fortiori if, in addition, the solution, A, is nonsingular. Conversely, if every eigenvalue of $U$ is less than $1 / 2$, then (14) possesses a solution, $A$ (indeed, the solution is unique) and this solution renders both sides of (14) positive definite.

To complete the proof of the Lemma, it suffices to show that among the open and nonempty set of parameter values for which the eigenvalues of $U$ are all less than $1 / 2$, the solution, $A$, to (14) is nonsingular for all but a closed subset having Lebesgue measure zero. Indeed, since the solution, B, to (A.1) satisfies $B=\Gamma A \Gamma^{T}$ where $\Gamma$ is nonsingular, it suffices to establish the following claim.

Claim: Restrict attention to the open and nonempty set of parameter values $\theta, \phi, \Sigma_{\eta}, \Sigma_{w}, \Sigma_{\varepsilon}$ such that all eigenvalues of $U$ are less than $1 / 2$. Then except possibly for a closed subset of parameter values having Labesgue measure zero, the solution, B, to (A.1) (which exists and is unique because the eigenvalues of $U$ are less than $1 / 2$ ) is nonsingular.

Proof of the Claim: The closedness result follows immediately from the fact that the solution, B, to 
(A.1) is continuous in the parameters when each eigenvalue is less than one-half. The measure zero result follows because, as is evident from (A.6) B can be written as $\mathrm{V}+\phi \mathrm{W}$, where $\mathrm{V}$ and $\mathrm{W}$ depend only upon $\theta, \Sigma_{\eta}, \Sigma_{w}$, and $\Sigma_{\varepsilon}$ but not $\phi$. Consequently, for each choice of $\theta, \Sigma_{\eta}, \Sigma_{w}$, and $\Sigma_{\varepsilon}$, B is singular for at most finitely many values of $\phi$, namely those that are zeros of the nondegenerate polynomial (in $\phi) \operatorname{det}(V+\phi W)$. To see that it is nondegenerate (ie. not identically equal to zero), it suffices to show that $V$ is nonsingular. But this follows because the $i j$ 'th entry of $V$ is, from (A.6), $\lambda_{i}\left(1-\lambda_{i}-\lambda_{j}\right)^{-1} f_{i j}$. Letting $f_{i j}^{*}=\left(1-\lambda_{i}-\lambda_{j}\right)^{-1} f_{i j}$ implies $F^{*}$ is positive definite. (Recall $F$ is positive definite, and the matrix $\left[\left(1-\lambda_{i}-\lambda_{j}\right)^{-1}\right]$ is positive semi-definite with no row or column of zeros. Then apply Lemma A.3.) Consequently, $V=\Lambda F^{*}$ so that $V^{-1}=F^{*-1} \Lambda^{-1}$. This completes the proof of the claim and the Lemma.

Q.E.D.

The remainder of this appendix is dedicated to the proof of Proposition 4.4. It relies on a number of Lemmas and we begin with these.

Lemma A.4: If $S_{0}$ is a peak, then $S_{0}$ is the unique utility maximizing demand for every insider-type in $\operatorname{ri\partial f}\left(S_{0}\right)$

Proof: Because $S_{0}$ is a peak, $m\left(\tau^{*}\right)=\left\{S_{0}\right\}$ for some insider type $\tau^{*}$. Hence,

$S_{0}^{T} \tau^{*}-f\left(S_{0}\right)>S^{T} \tau^{*}-f(S), \forall S \neq S_{0}$

Furthermore, by fact (d)(ii) of Appendix $B, \tau^{*} \in \partial f\left(S_{0}\right)$. Now since $\tau_{0} \in \operatorname{ri} \partial f\left(S_{0}\right)$ one may write it as

$\tau_{0}=\alpha \tau^{*}+(1-\alpha) \tau$, for some $\alpha \in(0,1]$ and some $\tau \in \partial f\left(S_{0}\right)$. Consequently, 


$$
\begin{aligned}
S_{0}^{T} \tau_{0}-F\left(S_{0}\right)= & S_{0}^{T} \tau_{0}-f\left(S_{0}\right), \\
& =\quad \alpha\left[S_{0}^{T} \tau^{*}-f\left(S_{0}\right)\right]+(1-\alpha)\left[S_{0}^{T} \tau-f\left(S_{0}\right)\right] \\
& >\quad \alpha\left[S^{T} \tau^{*}-f(S)\right]+(1-\alpha)\left[S^{T} \tau-f(S)\right] \forall S \neq S_{0}, \\
& =\quad S^{T_{1}} \tau_{0} f(S) \geq S^{T} \tau_{0}-F(S)
\end{aligned}
$$

where the first equality follows from fact (c)(ii) of Appendix B, from which one concludes that: $m\left(\tau^{*}\right)=\left\{S_{0}\right\}$ implies $F\left(S_{0}\right)=f\left(S_{0}\right)$. The inequality then derives from fact (d)(ii) of Appendix $B$, and $m\left(\tau^{*}\right)=\left\{S_{0}\right\}$. Finally, the last equality follows since by fact (b) of Appendix $B, f(s) \leq F(S)$. Q.E.D.

Define $h(S) \equiv U^{-1}\left[P(S)+\phi \Sigma_{0}(1-S)\right]$ for every S. Consequently, $U h(S)-P(S)-\phi \Sigma_{0}(1-S)=0$ for every $S$, and $h(S)$ has the following interpretation. The vector $h(S)$ is the point belief about the insider's type that would render 1-S a utility maximizing demand by each outsider subsequent to observing the insider demand $S$. Note that $h(\cdot)$ is simply a convenient technical device. We do not require the outsiders to form point beliefs.

In what follows, $\beta(S)$ denotes the outsiders' beliefs (probability measure) about the insider's type conditional on the insider's demand, $S$. To simplify the notation, we write only $\beta$ rather than $\beta(S)$.

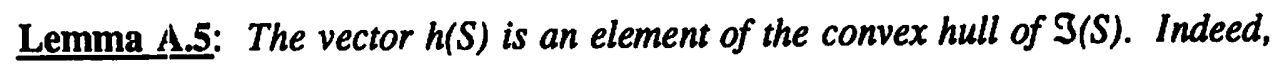

$$
h(S)=\frac{\int_{S(S)} \tau \cdot \psi(1-S, S, \tau) \beta(d \tau)}{\int_{S(S)} \psi(1-S, S, \tau) \beta(d \tau)}
$$

where $\psi(\mathrm{z}, \mathrm{S}, \tau)=\exp \left\{-\phi\left[\mathrm{z}^{\mathrm{T}} \mathrm{U} \tau-\mathrm{P}(\mathrm{S})^{\mathrm{T}} \mathrm{z}-\overline{\mathrm{S}}-.5 \phi \mathrm{z}^{\mathrm{T}} \Sigma_{\mathrm{o}} \mathrm{z}\right]\right\}$

Proof: From the well known properties of the exponential utility function and the normal distribution, conditional on any $\tau$, and any demands, $z$, by the outsider, and $S$ by the insider, one can write the 
outsider's utility as $\psi(z, S, \tau)=\exp \left\{-\phi\left[z^{T} U \tau-P(S)^{T} z-\bar{S}-.5 \phi z^{T} \Sigma_{0} z\right]\right\}$. The outsider's demand must therefore maximize, over $z$, the function $\int_{\mathfrak{S}(S)} \psi(z, S, \tau) \beta(d \tau)$. The resulting first-order condition is,

$$
\int_{\mathfrak{S}(S)}\left[U \tau-P(S)-\Sigma_{0} z\right] \psi(1-S, S, \tau) \beta(d \tau)=0
$$

Using the definition of $h(S)$ to substitute out $P(S)$ and rearranging proves (A.8). That $h(S)$ is in the convex hull of $\mathfrak{I}(S)$ then follows from the Corollary to Theorem 3 of Hildenbrand ([17], page 62). Q.E.D.

Lemma A.6: If $S_{0}$ is a peak, then $h\left(S_{0}\right) \in r i \partial f\left(S_{0}\right)$.

Proof: If $S_{0}$ is a peak, then by Lemma A.4, and Bayes' rule $\mathfrak{I}\left(S_{0}\right) \supseteq$ ridf $\left(S_{0}\right)$. But, since $\mathfrak{I}\left(S_{0}\right)$ is closed and by virtue of Proposition 4.3, one then has $\mathfrak{I}\left(\mathrm{S}_{0}\right)=\partial \mathrm{f}\left(\mathrm{S}_{0}\right)$.

Together with the previous Lemma this yields

$$
h\left(S_{0}\right)=\frac{\int_{\partial f\left(S_{0}\right)} \tau \psi\left(1-S_{0}, S_{0}, \tau\right) \beta(d \tau)}{\int_{\partial f\left(S_{0}\right)} \Psi\left(1-S_{0}, S_{0}, \tau\right) \beta(d \tau)}
$$

Since $\partial \mathrm{f}\left(\mathrm{S}_{0}\right)$ is convex, and the outsiders' prior belief (ie. before observing $S_{0}$ ) is normal, the measure $\mu$ defined by

$$
\mu(A)=\frac{\int_{\partial \cap \partial f\left(S_{0}\right)} \psi\left(1-S_{0}, S_{0}, \tau\right) \beta(d \tau)}{\int_{\partial r\left(S_{0}\right)} \psi\left(1-S_{0}, S_{0}, \tau\right) \beta(d \tau)}
$$

for every measurable subset $A$, gives full measure to ridf( $\left.\mathrm{S}_{0}\right)$. Hence, $\mathrm{h}\left(\mathrm{S}_{0}\right)=\int_{\partial \mathrm{f}\left(\mathrm{S}_{0}\right)} \tau \mu(\mathrm{d} \tau)=\int_{\mathrm{r} i \mathrm{f}\left(\mathrm{S}_{0}\right)} \tau \mu(\mathrm{d} \tau)$ so that by the Corollary to Theorem 3 of Hildenbrand ([17], page 
62), $h\left(S_{0}\right) \in \operatorname{ridf}\left(S_{0}\right)$. Q.E.D.

Lemma A.7: If $g: \boldsymbol{R}^{n} \rightarrow \boldsymbol{R}$ is convex, and $q^{f}$ is an extreme point of $\partial g\left(x_{0}\right)$, then there is a sequence $x_{m} \rightarrow x_{0}$ such that $\operatorname{pg}\left(x_{m}\right)=q_{m} \rightarrow q^{e}$.

Proof: In the course of proving Theorem 25.6 Rockafellar ([24], page 246) shows that every exposed point of $\partial g\left(x_{0}\right)$ is the limit of derivatives, $q_{m}=\sigma g\left(x_{m}\right)$, with $x_{m}$ converging to $x_{0}$. Combined with Straszewicz's Theorem (Rockafellar ([24], page 167)), which says that the set of exposed points of a closed convex set $\mathbf{C}$ is dense in the set of extreme points of $\mathrm{C}$, the proof is complete. Q.E.D.

Lemma A.8: If $S_{0}$ is a peak, then $\tau^{\mathrm{T}} \mathrm{U}\left(\mathrm{S}_{0}-\overline{\mathbf{S}}\right)=\mathrm{h}\left(\mathrm{S}_{0}\right)^{\mathrm{T}} \mathrm{U}\left(\mathbf{S}_{0}-\overline{\mathbf{S}}\right), \quad \forall \tau \in \partial \mathrm{f}\left(\mathrm{S}_{0}\right)$.

Proof: Choose any $\tau_{0} \in \operatorname{ridf}\left(S_{0}\right)$. By Lemma A.4 V( $\left.\tau_{0}\right)=S_{0}^{T} \tau_{0}-F\left(S_{0}\right)=S_{0}^{T} \tau_{0}-\left(S_{0}-\right.$ $\bar{S})^{\mathrm{T}} P\left(S_{0}\right)-.5 \theta S_{0}^{\mathrm{T}} \Sigma_{\eta} S_{0}$. So, by definition of h( $\left.S_{0}\right)$, $V\left(\tau_{0}\right)=S_{0}^{\mathrm{T}} \tau_{0}-\left(\mathrm{S}_{0}-\overline{\mathrm{S}}\right)^{\mathrm{T}}\left[\mathrm{Uh}\left(\mathrm{S}_{0}\right)-\phi \Sigma_{0}\left(1-\mathrm{S}_{0}\right)\right]-.5 \theta \mathrm{S}_{0}^{\mathrm{T}} \Sigma_{\eta} S_{0} . \quad$ By Lemma A.7, for any $\tau^{\mathrm{e}}$ extreme in $\partial \mathrm{f}\left(\mathrm{S}_{0}\right)$ there is a sequence $S_{m} \rightarrow S_{0}$, such that of $\left(S_{m}\right)=\tau_{m} \rightarrow \tau^{e}$. Therefore, by Proposition 4.3 and Lemma A.5, $h\left(S_{m}\right)=\tau_{m}$ for all $m$. Consequently, since $V\left(\tau_{0}\right) \geq S_{m}^{T} \tau_{0}-\left(S_{m}-\bar{S}\right)^{T}\left[U h\left(s_{m}\right)-\phi \Sigma_{0}\left(1-S_{m}\right)\right]-.5 \theta S_{m}^{T} \Sigma_{\eta} S_{m}$, we obtain upon taking the limit as $\mathrm{m} \rightarrow \infty$, that $\mathrm{h}\left(\mathrm{S}_{0}\right)^{\mathrm{T}} \mathrm{U}\left(\mathrm{S}_{0}-\overline{\mathrm{S}}\right) \leq\left(\tau^{\mathrm{e}}\right)^{\mathrm{T}} \mathrm{U}\left(\mathrm{S}_{0}-\overline{\mathrm{S}}\right)$, for all $\tau^{\mathrm{e}}$ extreme in $\partial \mathrm{f}\left(\mathrm{S}_{0}\right)$. But, by Lemma A.6 $h\left(S_{0}\right) \in \operatorname{ridf}\left(S_{0}\right)$. Hence, $h\left(S_{0}\right)^{T} U\left(S_{0}-\bar{S}\right)=\tau^{T} U\left(S_{0}-\bar{S}\right)$ for all $\tau \in \partial f\left(S_{0}\right)$. Q.E.D.

Proposition 4.4: In any equilibrium, every $S$ is a peak, and $F(\cdot$ is convex and equal to $f(\%$ Consequently, for almost every $S$, (i) the outsiders' beliefs are a point mass on $\nabla F(S)$, and (ii) $F(\cdot)$ satisfies the following differential equation: 


$$
F(S)=\sigma F(S)^{T} U(S-\bar{S})-\phi\left[S^{T} \Sigma_{0}(\bar{S}+1)-\bar{S}^{T} \Sigma_{0} 1\right]+S^{T}\left[\phi \Sigma_{0}+.5 \theta \Sigma_{\eta}\right] S,
$$

Proof: We first show that for every $\tau_{0}, \partial \mathrm{V}\left(\tau_{0}\right)$ is a singleton. So suppose by way of contradiction, that $\partial \mathrm{V}\left(\tau_{0}\right)$ contains two distinct points $S^{\mathrm{a}}$, and $S^{\mathrm{b}}$. Let $S^{\mathrm{ab}}=\alpha S^{\mathrm{a}}+(1-\alpha) S^{\mathrm{b}}, \tau^{*}=\mathrm{h}\left(S^{\mathrm{ab}}\right)$, and choose $\alpha \in(0,1)$ so that $S^{a b} \neq \bar{S}$. By Proposition 4.3 and Lemma A.5, $\tau^{*} \in \partial f\left(S^{a b}\right)$. Since by fact (d) of Appendix B both $S^{a}$ and $S^{b}$ maximize $S^{T} \tau_{0}-f(S)$, f must be linear on the line segment joining them. Fact (d)(ii) of Appendix B implies that $S^{a b}$ maximizes $S^{T} \tau^{*}-f(S)$. Consequently, the linearity of $f$ on the line segment joining $S^{a}$ and $S^{b}$ implies that both $S^{a}$ and $S^{b}$ maximize $S^{T} \tau^{*}-f(S)$. Hence $S^{a}$ and $S^{b}$ are contained in $\partial V\left(\tau^{*}\right)$, by facts (d)(i) and (d)(ii) of Appendix B.

Since $\partial \mathrm{V}\left(\tau^{*}\right)$ is compact and convex, the point $\mathrm{S}^{\mathrm{ab}}$, which is not extreme in $\partial \mathrm{V}\left(\tau^{*}\right)$, can be written as a strict convex combination of distinct extreme points of $\partial \mathrm{V}\left(\tau^{*}\right)$, say $S^{1}, \ldots, S^{k}$. By Lemma $A .7$, there exist sequences $\left\{\tau_{m}^{i}\right\}_{m=1}^{\infty}$ for every $i=1, \ldots, k$ such that $\tau_{m}^{i} \stackrel{m}{\rightarrow} \tau^{*}$ and $\nabla V\left(\tau_{m}^{i}\right)=S_{m}^{i} \rightarrow S^{i}$ for all $i$. So by fact (d) of Appendix $B, S_{m}^{i}$ is nice and $\tau_{m}^{i} \in \partial f\left(S_{m}^{i}\right)$ for all $i$, and $m$.

Let $Q(S)=\phi(S-\bar{S})^{T} \Sigma_{o}(1-S)-.5 \theta S^{T} \Sigma_{\eta} S$ for every S. Since $\phi \Sigma_{o}$ and $\theta \Sigma_{\eta}$ are positive definite, $Q$ is strictly concave. So for every $\mathrm{i}=1, \ldots \mathrm{k}$ we then have, by fact (d)(ii) of Appendix $\mathrm{B}$, that $\mathrm{V}\left(\tau_{m}^{\mathrm{i}}\right)=\left(\mathrm{S}_{\mathrm{m}}^{\mathrm{i}}\right)^{\mathrm{T}} \tau_{m}^{\mathbf{i}}-\mathrm{f}\left(S_{m}^{\mathrm{i}}\right)$. But because $S_{m}^{i}$ is a peak, fact (c) of Appendix $B$ then implies that $V\left(\tau_{m}^{i}\right)=\left(S_{m}^{i}\right)^{T} \tau_{m}^{i}-F\left(S_{m}^{i}\right)$. Using now both the definition of $F(\cdot)$ and $h(\cdot)$ to solve for $P(\cdot)$ yields $V\left(\tau_{m}^{i}\right)=\left(S_{m}^{i}\right)^{T} \tau_{m}^{i}-h\left(S_{m}^{i}\right)^{T} U\left(S_{m}^{i}-\bar{S}\right)+Q\left(S_{m}^{i}\right)$.

By Lemma A.8 one can rewrite the right-hand side to obtain $V\left(\tau_{m}^{i}\right)=\left(S_{m}^{i}\right)^{T} \tau_{m}^{i}-\left(\tau_{m}^{i}\right)^{T} U\left(S_{m}^{i}-\bar{S}\right)+Q\left(S_{m}^{i}\right)$ 


$$
\rightarrow \quad\left(S^{\mathbf{j}}\right)^{\mathbf{T}} \tau^{*}-\left(\tau^{*}\right)^{\mathbf{T}} U\left(\mathbf{S}^{\mathbf{i}}-\overline{\mathbf{S}}\right)+Q\left(\mathbf{S}^{\mathbf{i}}\right)
$$

However, $V\left(\tau_{m}^{i}\right) \rightarrow V\left(\tau^{*}\right)$ since $V(\cdot)$ is continuous. Hence,

$$
\begin{aligned}
& \mathrm{V}\left(\tau^{*}\right)=\quad\left(S^{\mathrm{j}}\right)^{\mathrm{T}} \tau^{*}-\left(\tau^{*}\right)^{\mathrm{T}} \mathrm{U}\left(S^{\mathrm{i}}-\overline{\mathrm{S}}\right)+\mathrm{Q}\left(S^{\mathrm{i}}\right), \forall \mathrm{i}=1, \ldots, \mathrm{k}, \text { and } \\
&<\quad\left(S^{\mathrm{ab}}\right)^{\mathrm{T}} \tau^{*}-\left(\tau^{*}\right)^{\mathrm{T}} \mathrm{U}\left(\mathrm{S}^{\mathrm{ab}}-\overline{\mathrm{S}}\right)+\mathrm{Q}\left(\mathrm{S}^{\mathrm{ab}}\right) \\
&=\left(S^{\mathrm{ab}}\right)^{\mathrm{T}} \tau^{*}-\mathrm{F}\left(S^{\mathrm{ab}}\right),
\end{aligned}
$$

where the inequality follows since $S^{\mathrm{ab}}$ is a strict convex combination of the distinct $S^{\mathrm{i}} \mathrm{s}$, and $Q(\cdot)$ is strictly concave; and where the second equality follows by virtue of $h\left(S^{\mathrm{ab}}\right)=\tau^{*}$ and the definitions of $\mathrm{F}(\cdot)$ and $\mathrm{h}(\cdot)$. Consequently, this implies that insider-type $\tau^{*}$ strictly prefers to demand $\mathrm{S}^{\mathrm{ab}}$ rather than demand his equilitrium quantity and obtain utility $\mathrm{V}\left(\tau^{*}\right)$. But this is a contradiction. Therefore, $\partial \mathrm{V}(\tau)$ is a singleton for every $\tau$.

We now show that $F(S)=f(S)$, so that by fact $(b)$ of Appendix $B, F(\cdot)$ is convex. Choose any $S$, and $\tau \in \partial f(S)$. Then by fact (d) of Appendix $B, S \in \partial V(\tau)=m(\tau)$. But $\partial V(\tau)$ is a singleton, so that $\{S\}=m(\tau)$. Consequently, by fact (c) of Appendix $B,\{S\}=m(\tau)=M(\tau)$ and $F(S)=f(S)$.

Next we show that every $S$ is a peak. Choose any $S_{0}$, and any $\tau_{0} \in f\left(S_{0}\right)$. Then by (d)(ii) of Appendix $B, S_{0} \in m\left(\tau_{0}\right)$. But this then implies that $M\left(\tau_{0}\right)=m\left(\tau_{0}\right)=\left\{S_{0}\right\}$. Hence, $S_{0}$ is a peak.

Being convex, $f$ (and hence $F$ ) is differentiable almost everywhere (Rockafellar [24], Theorem 25.5) so that by Proposition 4.3, the outsiders' beliefs conditional on $S$ are a point mass on $\nabla F(S)$ for almost every $S$.

Finally, we must derive the differential equation. But this has already been explained in the main text in the discussion following the statement of Proposition 4.4. Q.E.D. 


\section{Appendix B}

The following is a list of facts that are referred to in the text, all of which are taken from Rockafellar [24], denoted $[\mathbf{R}]$.

Facts:

(a) $V(\cdot)$ is convex. [R] Corollary 12.1 .1 , page 103.

(b) $f(\cdot)$ is well defined, convex and $f(S) \leq F(S)$ for all $S$. [R] Theorem 12.2, page 104.

(c) If $S \in M(\tau)$, then (i) $S \in m(\tau)$ and (ii) $f(s)=F(S)$. [R] Theorem 12.2, page 104. Consequently, for all $\tau$

$$
V(\tau)=\max _{S} S^{T} \tau-F(S)=\max _{S} S^{T} \tau-f(S)
$$

(d) (i) $m(\tau)=\partial V(\tau)$ for every $\tau$.

(ii) $S \in m(\tau)$ if and only if $\tau \in \partial f(S)$. [R] Theorem 23.5 page 218 . 


\section{REFERENCES}

1. G. Akerlof, The Market for Lemons: Qualitative Uncertainty and the Market Mechanism, Quart.J. Econ. 84 (1970), 488-500.

2. A. R. Admati, A Noisy Rational Expectations Equilibrium for Multi-Asset Securities Markets, Econometrica 53, (1985) 629-657.

3. L. M. Ausubel, Insider Trading in a Rational Expectations Economy, Amer. Econ. Rev. 80 (1990), 1022-1041.

4. J. Banks and J. Sobel, Equilibrium Selection in Signalling Games, Econometrica 55 (1987), 646-662.

5. M. Bray, Futures Trading, Rational Expectations, and the Efficient Market Hypothesis, Econometrica 49 (1981), 575-596.

6. U. Bhattacharya and M. Spiegel, Insiders, Outsiders and Market Breakdowns, Rev. Finan. Stud. 4 (1991), 25i5-282.

7. J. Caballe and M. Krishnan, Insider Trading and Asset Pricing in an Imperfectly Competitive Multisecurity Market, working paper, Purdue University (1989).

8. I. K. Cho and D. Kreps, Signalling Games and Stable Equilibria, Quart. J. Econ. 102 (1987), 179-221.

9. B. Chowdhry and V. Nanda, Multi-Market Trading and Market Liquidity, Rev. Finan. Stud. 4(3) (1991), 483-512.

10. D. Duffie and W. Shafer, Equilibrium in Incomplete Markets: I. A Basic Model of Generic Existence, J. Math. Econ. 14 (1985), 285-300.

11. D. Gale and M. F. Hellwig, Informed Speculation in Large Markets, working paper, University of Pittsburgh (1988).

12. L. R. Glosten, Insider Trading, Liquidity, and the Role of the Monopolist Specialist, J. Bus. 62 (1989), 211-235.

13. L. R.. Glosten and P. R. Milgrom Bid, Ask and Transaction Prices in a Specialist Model with Heterogerieously Informed Traders, J. Finan. Econ. 14 (1985), 71-100.

14. M. Fi. Hellwig, On The Aggregation of Information in Competitive Markets, J. Econ. Theory 22 (1980), 477-498.

15. M. F. Hellwig, Fully Revealing Outcomes in Signalling Models: An Example of Nonexistence when the Type Space is Unbounded, working paper, University of Basel (1991). 
16. R. A. Hom and C. R. Johnson, Topics in Matrix Analysis, Cambridge University Press, Cambridge (1991).

17. W. Hildenbrand, Core and Equilibria of a Large Economy, Princeton Studies in Mathematical Economics no. 5, Princeton University Press, Princeton, NJ (1974).

18. E. Kohlberg and J. F. Mertens, On the Strategic Stability of Equilibria, Econometrica 54 (1986), 1003-1037.

19. A. Kyle, Continuous Auctions and Insider Trading, Econometrica 53 (1985), 1315-1335.

20. J. C. Leach and A. N. Madhavan, Price Experimentation and Market Structure, working paper, University of Pennsylvania (1989).

21. J. J. Laffont and E. S. Maskin, The Efficient Market Hypothesis and Insider Trading on the Stock Market, J. Polit. Econ. 98 (1990), 70-93.

22. K. Mischel, H. M. Polemarchakis and P. Siconolfi, Noninformative Rational Expectations Equilibria when Assets are Nominal: An Example, Geneva Papers on Risk and Insurance Theory 15 (1990), 73-79.

23. R. Rahi, Partially Revealing Rational Expectations Equilibria with Nominal Assets, working paper, Stanford University, (1992).

24. R. T. Rockafellar, "Convex Analysis," Princeton University Press, Princeton NJ, 1970.

25. G. Strang, "Linear Algebra and its Applications," Academic Press Inc., New York NY, 1976. 


\section{Footnotes}

1.Our analysis also relates to that of Chowdhry and Nanda [9]. They present a Kyle type model in which a single sf:curity trades within different exchanges. The key feature of their model is that market makers do not observe prices on competing exchanges. Here there are several different securities which trade in a single market and their prices are common knowledge.

2.Since stability (Kohlberg and Mertens [18]), universal divinity (Banks and Sobel [4]) and Cho-Krep's never a weak best response criteria (Cho and Kreps [8]) all imply the D1 criterion, any of these refinements are compatible with our results.

3.Throughout the paper we maintain the following convention when the possibility of confusion might arise. Boldface refers to a function (or random variable) while normal face refers to the value taken on by the function (or random variable).

4.The status of our Walrasian market-maker is identical to that in traditional models of general competitive equilibrium. She is not an agent within the model, but merely a convenient expository device. Both Glosten and Milgrom [13] and Kyle [19] also employ a market-maker in this same spirit. Indeed, the market-efficiency condition they impose on their equilibrium price schedule can be derived from our market-clearing condition by setting $\phi=0$.

5.This is in rather sharp contrast to the purely competitive case in which (fully revealing) equilibria generically do exist (see, for instance Duffie and Shafer [10]). Indeed, when nominal assets are present in a competitive setting there are a plethora of non fully revealing equilibria as well (see Mischel, Polemarchakis and Siconolfi [22] and Rahi [23]).

6.Inferences resulting from equilibrium signals are disciplined by Bayes' rule.

7.Strictly speaking, this is not precisely the property which is associated with the D1 criterion. The difference lies in our use of every rather than some particular. Consequently, the difference renders our ultimate restriction even more compelling. In addition, our restriction is then formally weaker than the 
standard D1 criterion. Although this formal distinction is present, we've nonetheless chosen to use the same terminology. Of course, all our results hold a fortiori if one employs the standard D1 criterion. 8.The D1 criterion also requires that every member of $T$ can, in fact, by deviating to $S_{0}$, be made at least as well off by some price response of the market-maker as they would have been in equilibrium. But in our model this is almost always trivially the case since for any $S_{0} \neq \bar{S}$, any insider-type can be made arbitrarily well off by an appropriately chosen price. And the case in which $S_{0}=\bar{S}$ does not need to be considered for our purposes.

9.The support of a probability measure is the smallest closed subset assigned probability one.

10.The appropriate value of $b$ is $\left(I-U^{-1}\right)^{-1}\left(A^{T} \bar{S}+\phi U^{-1} \Sigma_{o} 1\right)$. Since, by Lemmas A.1 and A.2 of Appendix, A, $U$ is diagonalizable with eigenvalues between zero and one, and nonsingular, $\left(I-U^{-1}\right)^{-1}$ is well-defined. 11.Given any nxn, symmetric, positive definite matrix, one can perturb the $n(n+1) / 2$ elements along the diagonal and above in an arbitrary fashion while adjusting the elements below the diagonal to retain symmetry. If such a perturbation is slight enough, then the resulting symmetric matrix will also be positive definite. Consequently, the set of nxn, symmetric positive definite matrices is an open, nonempty, convex cone in $\mathbf{R}^{\mathrm{n}(\mathrm{n}+1) / 2}$. Likewise, the set of parameter values $\phi, \theta, \Sigma_{\eta}, \Sigma_{W}$, and $\Sigma_{\varepsilon}$ that are feasible in our model constitutes an open, nonempty, convex cone in $\mathbf{R}^{2+3 n(n+1) / 2}$. Accordingly, statements about openness, closedness, or Lebesgue measure are all with reference to the usual topology in $[2+3 n(n+1) / 2]-$ dimensional Euclidean space.

12. Note that because $P(\cdot)$ is an equilibrium price schedule, this maximization problem is well defined for every $\tau$.

13.See, for instance, Rockafellar ([24], pages 102-104). But note that $F(\cdot)$ need not, at this point, be convex. 
14.Appendix B contains several facts from convex analysis that we refer to throughout this part of the text.

These facts are labeled (a) through (d).

15.Note that the converse need not be true since $M(\tau)$ being a singleton does not imply that $m(\tau)$ is.

16.Note that this does not imply that $f(\cdot)$ is differentiable. For instance, consider the one variable function $f(S)=S^{2}$ for $S \geq 0$ and $f(S)=2 S^{2}$ for $S \leq 0$ and note that every $S$ is a peak for $f(\cdot)$. 


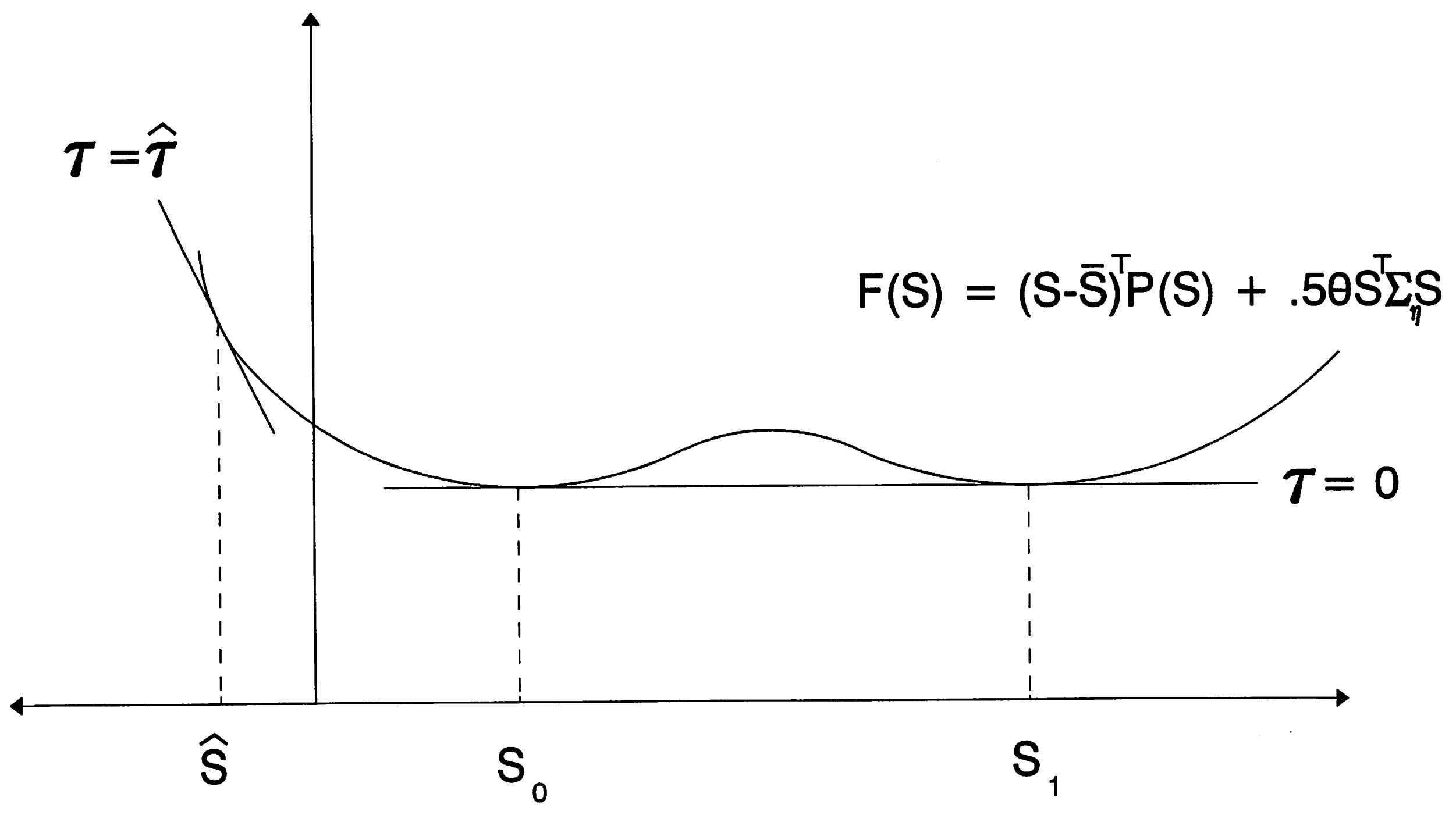

Figure 1 


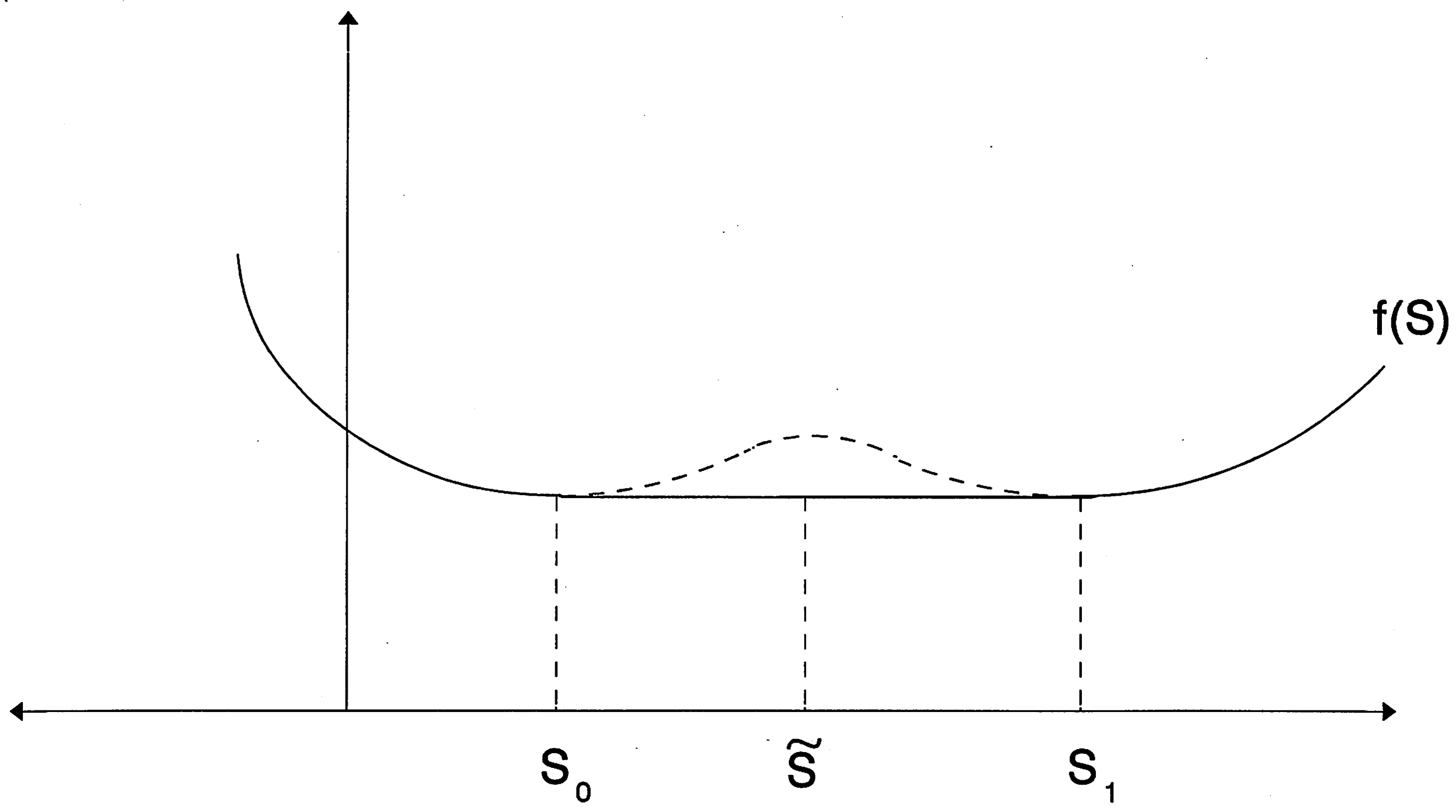

Figure 2 